\title{
Competitive Equilibrium with Search Frictions: a General Equilibrium Approach*
}

\author{
Belén Jerez ${ }^{\dagger}$
}

December, 2012

\begin{abstract}
When the trading process is characterized by search frictions, traders may be rationed so markets need not clear. We build a general equilibrium model with transferable utility where the uncertainty arising from rationing is incorporated in the definition of a commodity, in the spirit of the Arrow-Debreu theory. Prices of commodities then depend not only on their physical characteristics, but also on the probability that their trade is rationed. The standard definition of competitive equilibrium is extended by replacing market clearing with a matching condition which describes a trading technology that is not frictionless. This condition relates the rationing probabilities of buyers and sellers to ratio of buyers to sellers in the market via an exogenous matching function with constant returns, as in standard search-theoretic models. When search frictions vanish, our model is equivalent to the competitive assignment model of Gretsky, Ostroy and Zame (1992). We adopt their approach, which uses linear programming techniques and duality theory, to derive the welfare and existence theorems in our search environment. Our competitive equilibrium notion is equivalent to that of directed (or competitive) search. The strength of our formulation and the linear programming approach is that they allow us to generalize the constrained efficiency and existence results in the directed search literature to a much broader class of economies. Our framework also opens the door to the use of linear programming algorithms for computing equilibria.
\end{abstract}

Journal of Economic Literature Classification Numbers: D50, D61, D83.

Key Words: search frictions; transferable utility; competitive equilibrium; matching function; linear programming and duality; directed search.

"This draft has greatly benefited from the comments of several anonymous referees. I am grateful to Joe Ostroy for very helpful comments, and both to him and Louis Makowski for an insightful conversation that inspired this work. I also thank Jan Eeckhout, Onésimo Hernández, Philipp Kircher, Juan Pablo Rincón, Victor Ríos-Rull, Joon Song, and participants at the 2011 SAET Conference at Faro. This work started while I was visiting the University of Pennsylvania. I thank the Economics Department, and specially Victor Ríos-Rull, Kenn Burdett and Randy Wright, for their kind hospitality. Financial support from Spanish DGCYT (projects SEJ2004-07861, SEJ2007-65169, ECO2010-20614 and Ramón y Cajal Program), the Bank of Spain's Programme of Excellence in Research (2011-2013), and Fundación Ramón Areces is gratefully acknowledged. Any errors are mine.

\footnotetext{
† Departamento de Economía, Universidad Carlos III de Madrid, 28903 Getafe, Spain. E-mail: mjerez@eco.uc3m.es.
} 


\section{Introduction}

The Arrow-Debreu model is the cornerstone for the analysis of competitive markets. In the classical theory of Arrow (1964) and Debreu (1959), trade is represented as a costless process. Any agent seeking to buy or sell a good at a given point in time can do so at the equilibrium market price. Trade involves no further costs in terms of time and resources. Search theory, on the other hand, highlights the costly nature of the trading process. In particular, this theory has become the dominant paradigm to study labor markets since the seminal work of Diamond $(1981,1982)$, Mortensen (1982a, 1982b) and Pissarides (1984, 1985). In these markets workers usually take time and spend resources in order to find a suitable employer, and vice versa. Also, rationing arises in the form of unemployment, which tends to coexist with unfilled job vacancies. The key assumption of the Diamond-Mortensen-Pissarides model is that workers and firms must search for trading opportunities, and the outcome of their search is uncertain. Search frictions are typically modeled via an exogenous matching function which describes a random meeting process between workers and firms. ${ }^{1}$ This random process implies that at any point in time some agents will manage to trade and others will not. Hence, unlike in the classical Arrow-Debreu model, agents may be rationed in equilibrium (so markets need not clear), and in general it will take time to trade.

In this paper we study a prototypical class of exchange economies with search frictions and transferable utility, using a notion of competitive equilibrium in the spirit of Arrow and Debreu. ${ }^{2}$ We show that a competitive equilibrium exists and the two welfare theorems hold.

Our equilibrium notion is equivalent to that of directed (or competitive) search. ${ }^{3}$ It is wellknown that a directed search equilibrium is constrained efficient, both in simple environments with homogeneous buyers and sellers (see Moen 1997 and Shimer 1996) and in several environments with heterogeneity (e.g. Shi 2001, Shimer 2005 and Eeckhout and Kircher 2010). The point we want to make in this paper is that there is a well-established mathematical literature on infinitedimensional linear programming which has been used by Gretsky, Ostroy and Zame (1992, 1999) to study frictionless matching markets, and can be applied to study a relatively general search

\footnotetext{
${ }^{1}$ The matching function gives the measure of meetings between a worker and a firm as a function of the measures of workers and firms in the market, and possibly other variables such as the agents' search efforts.

${ }^{2}$ The transferable utility assumption is standard in the search literature.

${ }^{3}$ See Montgomery (1991), Peters (1991, 1997, 2000), Moen (1997), Acemoglu and Shimer (1999a, 1999b), Burdett, Shi, and Wright (2001), Shi (2001, 2009), Mortensen and Wright (2002), Shimer (2005), Menzio and Shi (2009, 2010) and Eeckhout and Kircher (2010), among others, as well as the survey by Rogerson, Shimer, and Wright (2005).
} 
environment. The strength of our competitive formulation and the linear programming approach is that it allows us to generalize the constrained efficiency and existence results in the directed search literature to a broader class of economies (e.g. with a large variety of different goods, a lot of diversity in buyer and seller tastes, with or without complementarities, possibly with heterogeneous matching technologies,...). ${ }^{4}$ This is accomplished by bringing to light a direct mapping between a competitive equilibrium and the solution to the social planner's problem. Our framework also opens the door to the use of existing linear programming algorithms for computing equilibria. Hence, it should be particularly useful for quantitative studies of search markets, where there is typically a lot of heterogeneity in prices and outcomes. ${ }^{5}$ To the best of our knowledge, the linear programming approach has not been used before to study economies with search frictions. ${ }^{6}$

Our key modeling choice is to incorporate the uncertainty arising from rationing in the definition of a commodity, as in the Arrow-Debreu theory. Prices of commodities then depend not only on their physical characteristics, but also on the probability that their trade is rationed (i.e., their "search characteristic"). In a competitive equilibrium agents - who are infinitesimal relative to the size of the economy - take prices as given. They also take as given rationing probabilities, which are part of the description of a commodity. Markets are anonymous, so prices and rationing probabilities do not depend on the identities of the traders. The departure from the standard definition of competitive equilibrium is that market clearing is replaced with a matching condition which describes a trading technology that is not frictionless. As in standard search-theoretic models, the matching condition relates the trading probabilities of buyers and sellers to the ratio of buyers to sellers in each market via an exogenous matching function with constant returns to scale. This condition says that the measures of buyers and sellers who trade a given commodity are equal (even though the measures of buyers and sellers in the market for the commodity may differ). In equilibrium, the price system adjusts so that the optimal decisions of the agents are consistent with the matching condition.

\footnotetext{
${ }^{4}$ This class, however, does not include the economies analyzed by Acemoglu and Shimer (1999a), who allow for risk aversion, and Shi (2009) and Menzio and Shi (2009), who study nonstationary infinite horizon dynamic models (see below). Neither does it include economies with adverse selection and moral hazard, like those studied by Guerrieri, Shimer, and Wright (2010) and Moen and Rosen (2011).

${ }^{5}$ In particular, it could be useful for the literature that focuses on structural estimation of hedonic models. Ekeland, Heckman, and Nesheim (2004) discuss the identification and estimation of the structure and the distribution of preferences and technologies in hedonic models without frictions using demand, supply and pricing data. We thank a referee for pointing this out to us.

${ }^{6}$ See Makowski and Ostroy $(1996,2003)$ for other general equilibrium formulations with transferable utility which use the linear programming approach. Jerez (2003), Rahman (2005) and Song (2012) adopt this approach to study competitive economies with adverse selection and moral hazard.
} 
The matching function captures the presence of external congestion effects in the trading process. Intuitively, as the ratio of buyers to sellers (or "market tightness") increases, the probability that each buyer trades falls and the probability that a seller trades increases. In other words, agents seeking to trade impose a negative congestion externality on traders on the same side of the market and a positive externality on traders on the other side of the market. In a competitive equilibrium these external effects are internalized, so the equilibrium allocation is constrained efficient. ${ }^{7}$ Because rationing probabilities are specified in the definition of a commodity, they are explicitly priced. Suppose, for instance, that the same physical good trades in two locations. Suppose also that there are fewer buyers per seller in the first location in equilibrium. If buyers and sellers are expected utility maximizers who are free to choose the location where they trade, the good should trade at a higher price in the first location (because there the probability of trading is higher for buyers and lower for sellers). The fact that the price of the good differs across locations is not surprising from the general equilibrium perspective, since the objects traded are formally two different commodities (described by their physical characteristics and the ratio of buyers to sellers at the trading location).

The standard definition of directed search equilibrium in this kind of environment assumes that sellers choose prices. Buyers in turn choose the good they want to buy and a price offer. Put differently, agents choose to trade in a particular "submarket," described by the good's physical characteristics and a price. All traders form beliefs about the ratio of buyers to sellers (and thus the probability of trading) in each submarket. The intuition is that, for a given good, lower prices attract relatively more buyers, increasing the sellers' trading probability and decreasing the buyers' trading probability. ${ }^{8}$ In equilibrium beliefs are rational in all active submarkets, meaning that the measures of buyers and sellers who trade in these submarkets generate the buyer-seller ratios that the traders take as given. On the other hand, beliefs in inactive submarkets are assumed to be always well-defined and common for all traders. ${ }^{9}$ Specifically, traders believe that there are many

\footnotetext{
${ }^{7}$ The definition of constrained efficiency takes into account the fact that the social planner, just like the market, is restricted by the matching technology.

${ }^{8}$ By contrasts, in the Diamond-Mortensen-Pissarides model each transaction between a buyer and a seller constitutes a bilateral monopoly. There search equilibria are typically constrained inefficient, unless the surplus is divided according to the bargaining rule that internalizes the search externalities (see Diamond 1982 and Hosios 1990).

${ }^{9}$ This formulation is closely related to Gale's (1996) general equilibrium model. See, for instance, Peters (1997). In Gale (1996) rationing arises from the presence of adverse selection (there are no search frictions). The objects of trade are contracts which provide a complete specification of the terms of trade, including prices. The price system then no longer plays the role of ensuring that individual decisions are consistent at the aggregate level. Instead it is the probabilities of trading the different contracts that play that role. Agents form common beliefs about these probabilities and the unobservable type of the traders they meet randomly in each market. In equilibrium trading probabilities are such that the long side of the market is rationed when there is an imbalance of buyers and sellers.
} 
buyers and sellers in all submarkets, even if they are inactive in equilibrium. Also, a particular restriction, known as the "market utility property", is imposed on beliefs. For environments where traders on one side of the market are homogeneous, the restriction implies that these traders are indifferent between active and inactive submarkets; i.e., they get their "market utility level" in all submarkets (see Peters (2000)). If, for instance, all buyers are homogeneous, one can then characterize the equilibrium by assuming that sellers choose a price and a ratio of buyers to sellers to maximize their expected utility subject to the constraint that buyers get their market utility level. Hence, the equilibrium is constrained efficient. Eeckhout and Kircher (2010) show that, with two-sided heterogeneity, the restriction is slightly more involved. It says that the buyer types who are most eager to trade in a given inactive submarket are indifferent between this submarket and the submarket where they trade in equilibrium. Alternatively, if all buyer types strictly prefer not to trade in the inactive submarket, agents believe that the tightness in this submarket is zero.

Peters $(1991,1997,2000)$ provides game theoretic foundations of directed search equilibrium for environments with one-sided heterogeneity (see also Burdett, Shi, and Wright (2001)). He considers a two-stage game, where some sellers first compete by simultaneously posting and committing to a price. Buyers then observe all the posted offers and direct their search to the most attractive ones. As the number of traders gets large, the equilibrium of the game coincides with a directed search equilibrium of a large search economy with an urn-ball matching function. In particular, in the limit game the conjectures of the sellers (who correctly anticipate the effects on buyers' behavior of making an off-the-equilibrium-path offer) have the market utility property.

Note that the two formulations are essentially two sides of the same coin. In directed search models agents choose a submarket (i.e., a good and a price) taking as given the ratio of buyers to sellers in all submarkets. In our model they choose a commodity (i.e., a good and a ratio of buyers to sellers) taking as given the prices of all commodities. As we shall see, our matching condition is the equivalent of the condition that beliefs are rational in all active markets. The equivalent of the assumption that all traders have the same beliefs in our model is that all traders face the same prices (prices are anonymous). And the equivalent of the assumption that beliefs in all markets (including inactive ones) are well-defined is that all commodities are priced, even if they are not traded in equilibrium. The latter feature is standard in general equilibrium models with a continuum

Also, beliefs are rational in all active markets, and in inactive markets they are pinned down using refinements analogous to those used in games of incomplete information. 
of commodities, like ours, where the prices of commodities which are not traded in equilibrium are indeterminate (e.g. see Mas-Colell and Zame 1991). The difference between the two formulations has to do with the market utility property. In our model prices in inactive markets keep buyers and sellers out of these markets in equilibrium, but need not provide any agent with her market utility. So our definition is in principle less restrictive. Yet a common convention is to get rid of the price indeterminacy by selecting the infimum of the set of supporting price systems (e.g. see Gretsky, Ostroy, and Zame 1999). We show that this is equivalent to the market utility assumption.

If the first contribution of this paper is to connect directed search models and the Arrow-Debreu model, the second and main contribution is technical. Our formulation is closer in spirit to classical general equilibrium theory in that it fleshes out the allocating role of prices. ${ }^{10}$ "Flipping things over" and laying out the search model in this more standard form is particularly useful for the following reason. In the absence of search frictions, our model is isomorphic to the competitive model of Gretsky, Ostroy, and Zame (1992). These authors analyze the linear assignment model of Shapley (1955) and Shapley and Shubik (1972) using linear programming techniques and duality theory. ${ }^{11}$ Our formulation purposely highlights the linear structure of the search environment, so it allows us to adopt a similar methodology to derive our results. The argument is the same as that of Gretsky, Ostroy, and Zame (1992), though our method of proof is different (mainly because, unlike in their paper, the measures describing an allocation are defined over a non-compact set). ${ }^{12}$ We first show that constrained efficient allocations solve a linear programming problem. Then we establish an equivalence between (i) the optimal solutions to this linear program and competitive equilibrium allocations, and (ii) the optimal solutions to the dual of the linear program and competitive equilibrium prices and indirect utilities (or market utility levels). The existence

\footnotetext{
${ }^{10}$ In this sense it is closer to the competitive search formulation in Mortensen and Wright (2002), which is similar to that in Moen (1997). These authors consider an economy with no complementarities and a single good (labor). In their model, the good may trade in different submarkets with different levels of market tightness which are explicitly priced. In equilibrium agents select the most preferred submarket taking as given the set of existing submarkets and the prices in these submarkets. Prices adjust so that the actual level of market tightness in each submarket equals the level that agents take as given. Finally, it is not possible to open additional submarkets that would attract positive measures of buyers and sellers. The interpretation is that there are third-party market makers with a profit motive who exploit existing arbitrage opportunities by setting up submarkets.

${ }^{11}$ Gretsky, Ostroy, and Zame (1999) use the same framework to clarify the concept of perfect competition.

${ }^{12}$ For this reason, our linear programming formulation (e.g. the choice of primal and dual spaces, and the corresponding topologies) is also slightly different. The same issue arises in Jerez (2003). The difference is that there the planner's problem is a linear semi-infinite program (e.g. with finitely many constraints), which allows to restrict to measures with finite support. Gretsky, Ostroy, and Zame (2002) derive conditions to guarantee existence of solutions to the dual program and equality of the values of the primal and dual programs for a general infinite-dimensional linear programming problem, but these are not applicable to our problem unfortunately.
} 
of a competitive equilibrium thus follows from the existence of optimal solutions to the primal and dual linear programs, under certain regularity conditions.

Our work contributes to a growing literature that has advanced methods which extend directed search to environments with a lot of heterogeneity. In particular, Eeckhout and Kircher (2010) study a similar class of search economies. They derive conditions for the equilibrium allocation to entail either positive or negative assortative matching. They also show that, under these conditions (which regard the degree of supermodularity of the match value function), an equilibrium exists and is constrained efficient. Our welfare and existence theorems generalize the latter results since they hold regardless of the matching pattern displayed by the equilibrium allocation. Also, they do not require the differentiability and concavity assumptions on utility and matching functions typically imposed in the literature. All we require is continuous utility and matching functions, and compact sets of physical goods and agents types.

Our framework embeds most static models in the directed search literature as particular cases, and extends easily to dynamic models which are stationary or have a finite horizon. Yet it does not apply to nonstationary infinite horizon dynamic models (see, however, Shi 2009 and Menzio and Shi 2009). This is because our proofs rely on the fact that one can restrict to a compact subset of the set of commodities without loss of generality (which is not true with an infinite horizon). We leave the analysis of this extension for future research.

The paper is organized as follows. Sections 2 and 3 describe the environment and the general equilibrium model, respectively. In Section 4 we define a competitive equilibrium and derive the welfare and existence theorems. In Section 5 we first illustrate the equivalence between a competitive equilibrium and a directed search equilibrium using examples. Then we present the equivalence result formally. The linear programming model and the main proofs are presented in the Appendix.

\section{The Economy}

There is a continuum of buyers and sellers of different types. Goods are indivisible and indexed by $h \in H$; so $H$ denotes the set of goods. Think of $h$ as a list of observable characteristics describing a good. In the labor market example, $h$ could be a list of features describing a job (e.g. sector, hours worked, tasks performed, location,...). In a housing market example, it could be a list of 
features describing a housing unit (e.g. size, number of bedrooms/bathrooms, neighborhood, nearby transportation, year of construction...). Goods are indivisible because agents cannot "perform half of job $h$ " or "buy half of housing unit $h$ ". They either perform the job (buy the unit) or not. Yet it is possible that the description of jobs $h^{\prime}$ and $h$ is identical, except that $h^{\prime}$ requires half of the hours worked. In our formulation, $h$ and $h^{\prime}$ are two different jobs (e.g. "full time" and "part time").

The set of seller types is $H$, meaning that the type of a seller is simply the type of good he supplies. ${ }^{13}$ The set of buyer types is denoted by $B$. We assume that $H$ and $B$ are compact metric spaces, and that there is a continuum of agents of each type. ${ }^{14}$ A particular case is that where $B$ and $H$ are finite sets. The population is described by a pair of Borel measures $\left(\xi^{\mathcal{B}}, \xi^{\mathcal{S}}\right) \in M_{+}(B) \times M_{+}(H)$ which characterize the subpopulations of buyers and sellers.

Each buyer wants to consume, and each seller can supply, at most one unit of a good. Agents have von Neumann-Morgernstern preferences and utility is transferable, so there is also a divisible numeraire good (available in positive and negative amounts) which yields equal constant marginal utility to all agents. ${ }^{15}$ Buyer valuations are described by the function $u: B \times H \rightarrow \Re_{+}$, where $u(b, h)$ is the value a type- $b$ buyer assigns to a unit of good $h$. Similarly, seller valuations are described by the function $v: H \rightarrow \Re_{+}$, where $v(h)$ is the cost or reservation value of a type- $h$ seller. In the labor market example, if a type- $b$ firm hires a type- $h$ worker then $u(b, h)$ is firm's output and $v(h)$ is the worker's disutility. In the housing market example, if a type- $b$ buyer purchases a type- $h$ unit, $u(b, h)$ is the buyer's valuation and $v(h)$ is the seller's reservation value for that unit. We assume that $u$ and $v$ are continuous. This assumption means not only that goods with similar features are perceived as similar by buyers and have similar costs, but also that similar buyer types have similar valuations for a given good. As in the Arrow-Debreu model, trader valuations are private information and all the relevant information is transmitted through the price system. We focus on the case where the gains from trading all goods are positive: $v(h)<\sup _{b \in B} u(b, h)$ for all $h \in H$.

So far our environment is identical to the continuous assignment model of Gretsky, Ostroy and Zame (1992). The key difference is that here the trading technology is not frictionless. In the

\footnotetext{
${ }^{13}$ That is, type- $h$ sellers are endowed with type- $h$ goods. In Jerez (2010) we consider a variant of the model where sellers choose the goods they sell. That paper assumes a finite number of goods and agent types. Yet a similar argument to the one used in this paper allows to derive the welfare and existence theorems in that setting with a continuum of goods and types. Ekeland (2010) studies the frictionless version of that model using convex analysis.

${ }^{14}$ As in Gretsky, Ostroy and Zame (1992), this is to avoid fractional assignments.

${ }^{15}$ These assumptions are standard in the search literature.
} 
labor market example, not all workers searching for jobs will find one, and the same is true for firms searching for workers. Also, some jobs may be harder to find/fill than others (just like certain housing units, say larger ones, may be harder to sell than others). In general, the probability that buyers and sellers are rationed will depend on the goods they seek to trade. Rationing probabilities, however, do not depend on the identity of the traders since markets are assumed anonymous.

Buyers and sellers who seek to trade a given good meet bilaterally and at random, as in standard search-theoretic models. The random meeting process is described by an exogenous matching function. To ease notation, we assume that the matching function is the same for all goods. Yet the results extend directly to the case where the matching function differs across goods. ${ }^{16}$ Suppose a measure $\beta$ of buyers and a measure $\sigma$ of sellers seek to trade a given good. The matching function $\mathcal{M}(\beta, \sigma)$ determines the total measure of bilateral matches. We assume that $\mathcal{M}: R_{+}^{2} \rightarrow R_{+}$is continuous, increasing, and homogeneous of degree one. Also, since the total number of matches cannot exceed the number of traders in the short side of the market, $\mathcal{M}(\beta, \sigma) \leq \min \{\beta, \sigma\}$. In particular, $\mathcal{M}(0, \sigma)=\mathcal{M}(\beta, 0)=0$. We assume that the Law of Large Numbers holds, so the probability that a seller meets a buyer is

$$
\pi(k)=\frac{\mathcal{M}(\beta, \sigma)}{\sigma}=\mathcal{M}(k, 1)
$$

where $k=\frac{\beta}{\sigma} \in \Re_{+}$is the expected number of buyers per seller (or market tightness). Likewise, the probability that a buyer meets a seller is

$$
\alpha(k)=\frac{\mathcal{M}(\beta, \sigma)}{\beta}=\mathcal{M}\left(1, k^{-1}\right)=\pi(k) k^{-1},
$$

with the convention that $\alpha(0)=\lim _{k \rightarrow 0} \alpha(k)$. The function $\pi(k)$ is then continuous and increasing, and $\alpha(k)$ is continuous and decreasing. It is customary to assume that $\pi(0)=0, \lim _{k \rightarrow \infty} \pi(k)=1$, $\alpha(0)=1$ and $\lim _{k \rightarrow \infty} \alpha(k)=0$. Intuitively, the higher the ratio of buyers to sellers, the easier it is for sellers to meet buyers and the harder it is for buyers to meet sellers. As $k$ goes to infinity (zero) the probability that a seller meets a buyer goes to one (zero), and the probability that a buyer meets a seller goes to zero (one). Note that $\alpha(k)$ and $\pi(k)$ also represent the fractions of buyers and sellers who meet a trading partner. Conversely, $1-\alpha(k)$ and $1-\pi(k)$ are the fractions of buyers

\footnotetext{
${ }^{16}$ Simply make the matching function specified below contingent on each $h \in H$, and denote it by $\mathcal{M}_{h}$. All we require is that $\mathcal{M}_{h}$ is continuous with respect to $h$, so the random meeting process is similar for similar goods.
} 
and sellers who are rationed.

\section{The General Equilibrium Model}

In this section, we define the commodity space and describe the set of feasible allocations.

\section{Commodities}

We describe commodities both by their physical characteristics and the uncertainty involved in their trade. Formally, a commodity is a pair $(h, k) \in H \times \Re_{+}$, where $h$ describes the physical good and $k$ is the ratio of buyers to sellers in a market for good $h$. As is standard, the numeraire good is not made explicit in the definition of a commodity.

\section{Allocations}

An allocation is an assignment of agents to commodities or to autarky (e.g. unemployment). To model the autarky choice it is convenient to introduce a real number $k_{0}<0$, and extend the probability functions in (2.1) and (2.2) to the set $K \equiv \Re_{+} \cup\left\{k_{0}\right\}$ by setting $\pi\left(k_{0}\right)=\alpha\left(k_{0}\right)=0$.

For a given a metric space $X$, let $M_{c}(X)$ be the space of signed regular Borel measures on $X$ with compact support, endowed with the weak-star topology. If $X$ is compact, this is just the space $M(X)$ of signed regular Borel measures on $X$. Following the measure-theoretic description in Hart, Hildenbrand, and Kohlberg (1974), an allocation is described by a pair of measures $\left(\mu^{\mathcal{B}}, \mu^{\mathcal{S}}\right) \in$ $M_{c+}(B \times H \times K) \times M_{c+}(H \times K)$. These measures are interpreted as follows. The assignment of buyers to commodities is described by the restriction of $\mu^{\mathcal{B}}$ to $B \times H \times \Re_{+}$, while their assignment to autarky is described by the restriction of $\mu^{\mathcal{B}}$ to the complement of this set. That is, for given Borel sets $E \subseteq B$ and $F \subseteq H \times \Re_{+}$, a measure $\mu^{\mathcal{B}}(E \times F)$ of buyers with types $b$ in $E$ are assigned

to a commodity $(h, k)$ in $F$, while a measure $\mu^{\mathcal{B}}\left(E \times H \times\left\{k_{0}\right\}\right)$ of these types are assigned to autarky. Sellers can only be assigned to commodity $(h, k)$ if they supply the corresponding good (i.e., if they are of type- $h$ ). The assignment of sellers to commodities and to autarky is described by the respective restrictions of $\mu^{\mathcal{S}}$ to $H \times \Re_{+}$and to the complement of this set. So, for given Borel sets $D \subseteq H$ and $G \subseteq \Re_{+}$, a measure $\mu^{\mathcal{S}}(D \times G)$ of sellers with types $h$ in $D$ are assigned to a commodity $(h, k)$ in $D \times G$, and a measure $\mu^{\mathcal{S}}\left(D \times\left\{k_{0}\right\}\right)$ of these types are assigned to autarky. Note that $\mu^{\mathcal{S}}(F)$ is the total measure of sellers assigned to a commodity $(h, k)$ in $F \subseteq H \times \Re_{+}$, or 
the aggregate supply of these commodities under the allocation.

It useful to define the marginals of $\mu^{\mathcal{B}}$ on $B$ and $H \times K$, which we denote by $\mu_{B}^{\mathcal{B}} \in M_{+}(B)$ and $\mu_{H \times K}^{\mathcal{B}} \in M_{c+}(H \times K)$. In particular, $\mu_{H \times K}^{\mathcal{B}}(F)$ is the total measure of buyers assigned to a commodity in $F \subseteq H \times \Re_{+}$, or the aggregate demand of these commodities under the allocation. Similarly, we define the marginal of $\mu^{\mathcal{S}}$ on $H, \mu_{H}^{\mathcal{S}} \in M_{+}(H)$. Finally, we denote the restrictions of $\mu_{H \times K}^{\mathcal{B}}$ and $\mu^{\mathcal{S}}$ to the set of commodities $H \times \Re_{+}$by $\tilde{\mu}_{H \times \Re_{+}}^{\mathcal{B}}$ and $\tilde{\mu}^{\mathcal{S}}$.

Feasible allocations must be consistent with respect to the population. This means that the total measure of buyers and sellers of a given type who are assigned to the different commodities or to autarky must be equal to the measure of such types who are present in the population (see also Gretsky, Ostroy, and Zame 1999, and Peters 1997):

$$
\begin{aligned}
& \mu_{B}^{\mathcal{B}}=\xi^{\mathcal{B}} \\
& \mu_{H}^{\mathcal{S}}=\xi^{\mathcal{S}} .
\end{aligned}
$$

Equivalently, $\mu_{B}^{\mathcal{B}}(E)=\xi^{\mathcal{B}}(E)$ and $\mu_{H}^{\mathcal{S}}(D)=\xi^{\mathcal{S}}(D)$ for all Borel sets $E \subseteq B$ and $D \subseteq H$.

Feasible allocations must also be consistent with respect to the matching technology. This requires that the measure of buyers who find a seller be equal to the measure of sellers who find a buyer for almost all commodities exchanged under these allocations:

$$
\int_{(h, k) \in F} \alpha(k) d \tilde{\mu}_{H \times \Re_{+}}^{\mathcal{B}}(h, k)=\int_{(h, k) \in F} \pi(k) d \tilde{\mu}^{\mathcal{S}}(h, k) \quad \text { for all Borel } F \subseteq H \times \Re_{+} .
$$

The measure of potential buyers of the different commodities is described by $\tilde{\mu}_{H \times \Re_{+}}^{\mathcal{B}}$. For each commodity $(h, k)$, the fraction of buyers who find a seller is $\alpha(k)$. Hence, the left-hand side of equation (3.3) is the measure of buyers of commodities in $F$ who find a seller. Similarly, the righthand side of (3.3) is the measure of sellers of these commodities who find a buyer. Condition (3.3) says that these two measures are equal for any Borel set $F$ of $H \times \Re_{+}$. 


\section{Competitive equilibrium}

In this section, we define a competitive equilibrium for the search economy and derive the welfare and existence theorems. We begin by describing the price space.

\section{Prices}

A price system is a non-negative continuous linear function on the set of commodities: ${ }^{17}$

$$
p \in C_{+}\left(H \times \Re_{+}\right),
$$

where $C_{+}\left(H \times \Re_{+}\right)$is endowed with the topology of uniform convergence on compact sets (see the Appendix). That is, $p(h, k) \in \Re_{+}$is the price of commodity $(h, k)$; i.e., the price of good $h$ when the ratio of buyers to sellers is equal to $k$. Prices then depend both on the goods' physical characteristics and their associated rationing probabilities. This is intuitive. If the same physical good is trades in two markets where the ratio of buyers to sellers is different, the price of the good may differ in the two markets. Formally, $(h, k)$ and $\left(h, k^{\prime}\right)$ are two different commodities when $k \neq k^{\prime}$, so $p(h, k)$ need not be equal to $p\left(h, k^{\prime}\right)$. On the other hand, since $p$ is continuous, similar commodities have similar prices. In particular, if the same physical good trades in two markets where the ratio of buyers to sellers is similar, the price of the good will be similar in both markets. It is convenient to extend prices so $p\left(h, k_{0}\right)=0$ for all $h$ in $H$; that is, the price associated to the autarky choice is zero.

\section{Competitive equilibrium}

We are now ready to define a competitive equilibrium. The key assumption is that agents have rational expectations about $k$, and hence about the probability with which they will be rationed when they choose to trade commodity $(h, k)$. The expected utility from commodity $(h, k)$ for a type- $b$ buyer is $\alpha(k)[u(b, h)-p(h, k)]$. In words, the buyer trades with probability $\alpha(k)$, in which case she gets her valuation $u(b, h)$ net of the market price $p(h, k)$. The buyer's expected utility then increases with the trading probability (decreases with $k$ ) and decreases with the price. Similarly, the expected utility of a type- $h$ seller is $\pi(k)[p(h, k)-v(h)]$, so it increases with both $k$ and the price. Autarky yields zero expected utility to all agents; i.e., $\alpha\left(k_{0}\right)\left[u(b, h)-p\left(h, k_{0}\right)\right]=0$ and

\footnotetext{
${ }^{17}$ We follow Mas-Colell's (1975) description of the price space for economies with a continuum of differentiated commodities. The difference is that, whereas in his model agents can trade any integer number of units of a given good, here agents trade at most one unit.
} 
$\pi\left(k_{0}\right)\left[p\left(h, k_{0}\right)-v(h)\right]=0$.

Definition 1. A competitive equilibrium for the search economy is an allocation $\left(\mu^{\mathcal{B} *}, \mu^{\mathcal{S} *}\right) \in M_{c+}(B \times$ $H \times K) \times M_{c+}(H \times K)$ and a price system $p^{*} \in C_{+}(H \times K)$ such that:

(i) For each type $b \in B$, buyers choose to trade a commodity that maximizes their expected utility taking $p^{*}$ as given:

$$
v_{b}^{*}\left(p^{*}\right) \equiv \sup _{(h, k) \in H \times K} \alpha(k)\left[u(b, h)-p^{*}(h, k)\right]=\alpha\left(k^{*}\right)\left[u\left(b, h^{*}\right)-p^{*}\left(h^{*}, k^{*}\right)\right],
$$

for almost all $\left(b, h^{*}, k^{*}\right) \in \operatorname{supp} \mu^{\mathcal{B} *}$.

(ii) For each type $h \in H$, sellers choose to trade a commodity that maximizes their expected utility taking $p^{*}$ as given:

$$
v_{h}^{*}\left(p^{*}\right) \equiv \sup _{k \in K} \pi(k)\left[p^{*}(h, k)-v(h)\right]=\pi\left(k^{*}\right)\left[p^{*}\left(h, k^{*}\right)-v(h)\right]
$$

for almost all $\left(h, k^{*}\right) \in \operatorname{supp} \mu^{\mathcal{S} *}$.

(iii) $\left(\mu^{\mathcal{B} *}, \mu^{\mathcal{S} *}\right)$ is consistent with the population:

$$
\mu_{B}^{\mathcal{B}}=\xi^{\mathcal{B}}, \text { and } \mu_{H}^{\mathcal{S}}=\xi^{\mathcal{S}}
$$

(iv) $\left(\mu^{\mathcal{B} *}, \mu^{\mathcal{S} *}\right)$ is consistent with the matching technology:

$$
\int_{(h, k) \in F} \alpha(k) d \tilde{\mu}_{H \times \Re_{+}}^{\mathcal{B}}(h, k)=\int_{(h, k) \in F} \pi(k) d \tilde{\mu}^{\mathcal{S}}(h, k) \text { for all Borel } F \subseteq H \times \Re_{+} .
$$

Conditions (i) and (ii) require that almost all buyers and sellers choose to trade a commodity that maximizes their expected utility at the equilibrium prices $p^{*}$. Note that sellers can only choose commodity $(h, k)$ if they are of type $h$. The expected indirect utility attained by type- $i$ agents in equilibrium is denoted by $v_{i}^{*}\left(p^{*}\right)$ for each $i \in B \cup H$. Condition (iii) ensures that the allocation is consistent with the population. The difference with respect to the standard definition of a competitive equilibrium is that, in condition (iv), market clearing has been replaced with the matching condition (3.3). 
In the absence of search frictions, market clearing would require that the measures of buyers and sellers be equal in almost all markets:

$$
\tilde{\mu}_{H \times \Re+}^{\mathcal{B}}=\tilde{\mu}^{\mathcal{S}} .
$$

Equivalently, $\tilde{\mu}_{H \times \Re_{+}}^{\mathcal{B}}(F)=\tilde{\mu}^{\mathcal{S}}(F)$ for all Borel sets $F \subseteq H \times \Re_{+}$. In this case, there is no rationing for almost all goods which are traded in equilibrium. Indeed, when the matching condition in (iv) is replaced by (4.6), our model is isomorphic to Gretsky, Ostroy and Zame's (1992).

The matching condition in (iv) can also be expressed as

$$
\int_{(h, k) \in F} d \tilde{\mu}_{H \times \Re_{+}}^{\mathcal{B}}(h, k)=\int_{(h, k) \in F} k d \tilde{\mu}^{\mathcal{S}}(h, k) \text { for all Borel } F \subseteq H \times \Re_{+},
$$

using (2.2), since $\alpha(k)>0$ for all $k \in \Re_{+}$. Equation (4.7) says that $\tilde{\mu}_{H \times \Re_{+}}^{\mathcal{B}}$ must be absolutely continuous with respect to $\tilde{\mu}^{\mathcal{S}}$ with Radon-Nikodym derivative $f(h, k)=k$. In words, the measures of buyers and sellers who choose to trade in almost every active market in equilibrium generate the buyer-seller ratios that these traders take as given when they choose to participate in those markets. ${ }^{18}$ So, as in directed search models, beliefs are rational in all active markets.

\section{Welfare Theorems and Existence}

In this economy, the planner is restricted by the exogenous matching technology that brings buyers and sellers together in the market. That is, the planner can choose an assignment of agents to commodities or to autarky, but cannot choose an assignment of buyers to sellers of these commodities as in Gretsky, Ostroy and Zame (1992). The appropriate efficiency notion is then that of constrained efficiency.

The total gains from trade from an allocation are

$$
\int_{B \times H \times K} \alpha(k) u(b, h) d \mu^{\mathcal{B}}(b, h, k)-\int_{H \times K} \pi(k) v(h) d \mu^{\mathcal{S}}(h, k) .
$$

The first term is the sum of the buyers' valuations for the physical goods assigned to them under the allocation. Remember that $\mu^{\mathcal{B}}$ describes the measure of buyers of each type $b$ who are assigned

\footnotetext{
${ }^{18}$ This is precisely Peters' (1997) generalized market clearing condition, which is the equivalent of Gale's (1996) condition of fulfilled expectations in active markets. See also Eeckhout and Kircher (2010).
} 
to each commodity $(h, k)$. A fraction $\alpha(k)$ of these buyers manage to trade, so they receive their valuation $u(b, h)$ (while the rest are rationed). Similarly, the second term is the sum of the sellers' reservation values for the goods sold under the allocation. That is, $\mu^{\mathcal{S}}$ describes the measure of sellers assigned to each commodity $(h, k)$, and only a fraction $\pi(k)$ of these sellers trade, at cost $v(h)$. The difference between the first and second term in (4.8) then gives the total gains from trade.

The planner's problem is to choose an allocation that maximizes (4.8) subject to the feasibility constraints (3.1)-(3.3). Note that the objective function and the constraints are linear in $\left(\mu^{\mathcal{B}}, \mu^{\mathcal{S}}\right)$ (they are integrals with respect to the allocation). So, as in Gretsky, Ostroy and Zame (1992), the planner's problem is an infinite dimensional linear programming problem. In the Appendix we show that the dual variables (or Lagrange multipliers) associated with constraints (3.1) and (3.2) are given by two continuous functions, $q^{\mathcal{B}} \in C(B)$ and $q^{\mathcal{S}} \in C(H)$. The optimal values of $q^{\mathcal{B}}(b)$ and $q^{\mathcal{S}}(s)$ give the expected utility of type- $b$ buyers and type-s sellers at a constrained efficient allocation. As we shall see, feasible dual solutions must satisfy $q^{\mathcal{B}}, q^{\mathcal{S}} \geq 0$, so individual rationality holds (see Lemma A.2). ${ }^{19}$ The dual variable associated with (3.3) is also given by a continuous function, $\lambda \in C(H \times K)$, and the optimal value of $\lambda(h, k)$ measures the shadow price of a unit of commodity $(h, k)$ at a constrained efficient allocation. We show that $\lambda \geq 0$ without loss of generality.

In the Appendix we exploit this linear structure to prove all the results that follow. The first result is the existence of optimal solutions to the planner's problem and its dual, and the absence of a "duality gap".

Theorem 1. The planner's linear programming problem has optimal solutions, and so does the dual of this problem. Moreover, the value of the two problems is the same (there is no "duality gap").

We also establish an equivalence between (i) the optimal solutions to the planner's problem and competitive equilibrium allocations, and (ii) the optimal solutions to the dual of the planner's problem and competitive equilibrium prices and indirect utilities (see Theorem A.5). Hence, the First and Second Welfare Theorems hold.

In fact, the First Welfare Theorem can be proved using a variation of the standard argument.

Theorem 2. A competitive equilibrium allocation $\left(\mu^{\mathcal{B} *}, \mu^{\mathcal{S} *}\right)$ is constrained efficient.

\footnotetext{
${ }^{19}$ This is a general property of the linear programming formulation of Gretsky, Ostroy and Zame (1992,1999) and Makowski and Ostroy (1996, 2003), where individual rationality constraints appear in the dual of the planner's problem (rather than in the primal problem).
} 
Proof. By conditions (iii) and (iv), a competitive equilibrium allocation a feasible solution to the planner's problem. Condition (i) implies that, for almost all $\left(b, h^{*}, k^{*}\right)$ in the support of $\mu^{\mathcal{B} *}$,

$$
\alpha\left(k^{*}\right)\left[u\left(b, h^{*}\right)-p^{*}\left(h^{*}, k^{*}\right)\right] \geq \alpha(k)\left[u(b, h)-p^{*}(h, k)\right] \text { for all }(h, k) \in H \times K .
$$

Similarly, condition (ii) implies that, for almost all $\left(h, k^{*}\right)$ in the support of $\mu^{\mathcal{S} *}$,

$$
\pi\left(k^{*}\right)\left[p^{*}\left(h, k^{*}\right)-v(h)\right] \geq \pi(k)\left[p^{*}(h, k)-v(h)\right] \text { for all } k \in K
$$

But then, for any other feasible allocation $\left(\mu^{\mathcal{B}}, \mu^{\mathcal{S}}\right)$,

$$
\begin{aligned}
& \int_{B \times H \times K} \alpha(k)\left[u(b, h)-p^{*}(h, k)\right] d \mu^{\mathcal{B} *}(b, h, k)+\int_{H \times K} \pi(k)\left[p^{*}(h, k)-v(h)\right] d \mu^{\mathcal{S} *}(h, k) \\
& \geq \int_{B \times H \times K} \alpha(k)\left[u(b, h)-p^{*}(h, k)\right] d \mu^{\mathcal{B}}(b, h, k)+\int_{H \times K} \pi(k)\left[p^{*}(h, k)-v(h)\right] d \mu^{\mathcal{S}}(h, k) .
\end{aligned}
$$

That is, total expected utility is at least as high under allocation $\left(\mu^{\mathcal{B} *}, \mu^{\mathcal{S} *}\right)$ than under allocation $\left(\mu^{\mathcal{B}}, \mu^{\mathcal{S}}\right)$. Rearranging this expression yields

$$
\begin{aligned}
& \int_{B \times H \times K} \alpha(k) u(b, h) d \mu^{\mathcal{B} *}(b, h, k)-\int_{H \times K} \pi(k) v(h) d \mu^{\mathcal{S} *}(h, k) \\
& -\int_{H \times \Re_{+}} \alpha(k) p^{*}(h, k) d \tilde{\mu}_{H \times \Re_{+}}^{\mathcal{B} *}(h, k)+\int_{H \times \Re_{+}} \pi(k) p^{*}(h, k) d \tilde{\mu}^{\mathcal{S} *}(h, k) \\
& \geq \int_{B \times H \times K} \alpha(k) u(b, h) d \mu^{\mathcal{B}}(b, h, k)-\int_{H \times K} \pi(k) v(h) d \mu^{\mathcal{S}}(h, k) \\
& -\int_{H \times \Re_{+}} \alpha(k) p^{*}(h, k) d \tilde{\mu}_{H \times \Re_{+}}^{\mathcal{B}}(h, k)+\int_{H \times \Re_{+}} \pi(k) p^{*}(h, k) d \tilde{\mu}^{\mathcal{S}}(h, k),
\end{aligned}
$$

since $\pi\left(k_{0}\right)=\alpha\left(k_{0}\right)=0$. Substituting condition (iv) expressed as in (4.7), and using (2.2) yields

$$
\begin{aligned}
& \int_{B \times H \times K} \alpha(k) u(b, h) d \mu^{\mathcal{B} *}(b, h, k)-\int_{H \times K} \pi(k) v^{s}(h) d \mu^{\mathcal{S} *}(h, k) \\
& \geq \int_{B \times H \times K} \alpha(k) u(b, h) d \mu^{\mathcal{B}}(b, h, k)-\int_{H \times K} \pi(k) v^{s}(h) d \mu^{\mathcal{S}}(h, k),
\end{aligned}
$$

since prices are just transfers which cancel out. Hence, $\left(\mu^{\mathcal{B} *}, \mu^{\mathcal{S} *}\right)$ solves the planner's problem.

The Second Welfare Theorem says that any optimal solution to the planner's problem can be attained as a competitive equilibrium. 
Theorem 3. Let $\left(\mu^{\mathcal{B}}, \mu^{\mathcal{S}}\right)$ be an optimal solution to the planner's problem. Then there is a price system $p \in C_{+}(H \times K)$ such that $\left(\mu^{\mathcal{B}}, \mu^{\mathcal{S}}, p\right)$ is a competitive equilibrium.

Finally, the existence of a competitive equilibrium follows from Theorem 1.

Theorem 4. A competitive equilibrium exists.

As shown in the Appendix, the proofs of Theorems 1, 3 and 4 rely on the assumptions that the valuation functions $u$ and $v$ and the matching function $\mathcal{M}$ are continuous, and $B$ and $H$ are compact sets. This, combined with the fact that trading probabilities are bounded, is all we need to prove these results.

\section{Relation with the Directed Search Literature}

In this section we discuss the connection between the competitive equilibrium notion in this paper and that of directed search. We first illustrate this connection using examples (which should be particularly useful for general equilibrium theorists who are not familiar with the directed search literature). Then we present the equivalence result formally.

Example 1. Homogeneous buyers and sellers. Take the simplest environment with a single good and homogeneous buyers (e.g. Moen 1997). There is a measure $\hat{\beta}$ of buyers and a measure $\hat{\sigma}$ of sellers, so the economy-wide market tightness is $\hat{\beta} / \hat{\sigma} .{ }^{20}$ Buyer valuations are denoted by $u>0$, and seller valuations are normalized to zero. In addition to the properties described in Section 2, the matching function $\mathcal{M}$ is strictly increasing, strictly concave and twice continuously differentiable.

In a directed search equilibrium, each trader chooses a price $p$ specifying the division of the surplus in a bilateral transaction between a buyer and a seller. Since buyers and sellers have the option of not trading, $p \in[0, u]$. The expected payoff to a seller who chooses price $p$ is $\pi(k) p$, where $k$ is the ratio of buyers to sellers associated to $p$, whereas the expected payoff to a buyer who seeks to trade at price $p$ is $\alpha(k)(u-p)$. All traders make optimal decisions based on their common beliefs about the relationship between $p$ and $k$. Also, these beliefs, denoted by $k(p)$, are rational.

\footnotetext{
${ }^{20}$ In Moen (1997) there is free entry of buyers (firms), so $\hat{\beta}$ is endogenous and its value is pinned down by a zero profit constraint on the buyers' equilibrium expected payoff characterized below.
} 
A directed search equilibrium is characterized as follows. Let $\Pi^{*}$ denote the set of prices at which buyers and sellers trade in equilibrium. Since buyers are homogeneous, they must get a common expected payoff $U^{*}$ in equilibrium - their "market utility" level. That is,

$$
\alpha\left(k^{*}\right)\left(u-p^{*}\right)=U^{*} \geq 0, \text { for all } p^{*} \in \Pi^{*},
$$

where $k^{*}=k\left(p^{*}\right)$. The traders' equilibrium beliefs $k^{*}(p)$ then satisfy (5.1) for $p \in \Pi^{*}$. The market utility assumption pins down beliefs for $p \notin \Pi^{*}$. Under this assumption, $k^{*}(p)$ satisfies (5.1) for all $p \in[0, u]$. That is, $k^{*}(p)$ coincides with the buyers' indifference curve associated to $U^{*}$ on the space $(p, k)$. The idea is that sellers take the buyers' market utility level as given, since they are infinitesimal relative to the size of the market. So, if a negligible mass of sellers deviates and chooses $p^{\prime} \notin \Pi^{*}$, they will assume that the deviation has no impact on the buyers' expected payoff. In particular, their beliefs about $k\left(p^{\prime}\right)$ would be based on the assumption that buyers always modify their decisions in a way that ensures that they are indifferent between the equilibrium price and $p^{\prime}$.

The above implies that each price $p^{*} \in \Pi^{*}$ and the associated buyer-seller ratio $k^{*}$ must maximize the sellers' expected payoff subject to the constraint that buyers get their market utility level $U^{*}$. So a directed search equilibrium is constrained efficient. The properties of the matching function imply that this convex problem has a unique solution. Hence, in equilibrium all agents choose the same price $p^{*}$ and, since beliefs are rational, $k^{*}=\hat{\beta} / \hat{\sigma}$. Substituting $p^{*}$ and $k^{*}$ into (5.1) we obtain the equilibrium value of $U^{*}$. As shown in Figure 1, an interior solution is characterized by the tangency between the indifference curves of the buyer and the seller on the $(k, p)$ space. This tangency condition is the well-known Hosios (1990) condition. $^{21}$

It is direct to see that the above outcome is attained in a competitive equilibrium. In this economy with a single physical good, a commodity is described by a level of market tightness $k \in \Re_{+}$. A price system is a continuous function $p(k)$, extended so $p\left(k_{0}\right)=0$. In Figure 1 we can see that any continuous function lying between the indifference curves of the buyer and the seller which is tangent to both curves at $\left(\hat{\beta} / \hat{\sigma}, p^{*}\right)$ supports the constrained efficient allocation. In

\footnotetext{
${ }^{21}$ Substituting $\alpha(k)=\pi(k) / k$ into the tangency condition, and rearranging yields the Hosios condition:

$$
\frac{u-p}{p}=\frac{\eta(k)}{1-\eta(k)} \text { where } \eta(k)=\frac{\pi^{\prime}(k) k}{\pi(k)} .
$$

This condition says that the buyers' share of the surplus is equal to the elasticity of the sellers' trading probability. Hence, $p^{*}$ solves (5.2) when $k=\hat{\beta} / \hat{\sigma}$. Note that, if $p^{*} \notin[0, u]$, the problem has a corner solution which is easily characterized (either the buyers or the sellers get a zero expected payoff).
} 
particular, $p(k)$ is indeterminate for $k \neq \hat{\beta} / \hat{\sigma}{ }^{22}$ As noted in the introduction, a common convention is to select the infimum of the set of supporting price systems. Figure 1 shows that the selected price system $p^{*}(k)$ coincides with the buyer's indifference curve, so it is the inverse of the function $k^{*}(p)$ specifying the equilibrium beliefs in a directed search equilibrium. ${ }^{23}$

The argument is essentially the same with heterogeneous sellers (and is symmetric with heterogeneous buyers). In a directed search equilibrium, one can think of each seller type as choosing $p$ and $k$ to maximize her expected payoff subject to the constraint that buyers get their market utility level (see Peters 2000). So the function describing the equilibrium beliefs again coincides with the buyers' indifference curve. It is also easy to see that the inverse of this function is the infimum of the set of supporting price systems in our model.

Example 2. Two-sided heterogeneity. Consider now an economy with two goods, $h_{1}$ and $h_{2}$, and two buyer types, $b_{1}$ and $b_{2}$. There is a measure $\hat{\beta}_{i}$ of type- $i$ buyers for $i=1,2$, and an equal measure $\hat{\sigma}$ of sellers of each good. All sellers have identical reservation values, normalized to zero. Also, buyer valuations satisfy $u\left(b_{1}, h_{1}\right)=u\left(b_{2}, h_{2}\right)=u_{H}$ and $u\left(b_{1}, h_{2}\right)=u\left(b_{2}, h_{1}\right)=u_{L}$, where $u_{H}>u_{L}>0$. Say $h_{1}$ are "red" houses (e.g. apartments facing south) and $h_{2}$ are "blue" houses (e.g. apartments facing north). Type-1 buyers prefer the former (which are warmer in the winter) and type-2 buyers prefer the latter (which are cooler in the summer). ${ }^{24}$

Environments with two-sided heterogeneity are more involved because one needs to characterize the matching pattern that emerges in equilibrium; i.e., which buyer types trade with each seller type. Potentially a given buyer type may trade with more than one seller type, and vice versa. ${ }^{25}$ Once we know the matching pattern, the appropriate tangency (e.g. Hosios) condition for each buyer-seller match together with the rationality of equilibrium beliefs will determine the equilibrium outcome essentially as in the previous example. As noted by Shi (2001) and Eeckhout and Kircher (2010), the Hosios condition is only necessary but not sufficient to characterize a directed search equilibrium with two-sided heterogeneity.

As discussed in Eeckhout and Kircher (2010), the market utility assumption is slightly more

\footnotetext{
${ }^{22}$ A related issue arises in Peters (1997), where equilibrium beliefs are indeterminate.

${ }^{23}$ Since the matching function is continuous and strictly monotone, so is $k^{*}(p)$. This function is then invertible and its inverse is the continuous function $p^{*}(k)$, and conversely.

${ }^{24}$ Here differences in buyer valuations are purely idiosyncratic. Shi (2001) and Eeckhout and Kircher (2008) present related examples with complementarities where valuations have a common value component.

${ }^{25}$ See Shi (2001) for an environment which rules this out.
} 
involved with two-sided heterogeneity. It says that the ratio of buyers to sellers in each inactive submarket is determined by the indifference condition of the buyer type who is most eager to trade there. Alternatively, if all buyer types strictly prefer not to trade in an inactive submarket, the ratio of buyers to sellers is zero. The authors show that one can then think of sellers as choosing a price, a buyer-seller ratio, and the buyer type they trade with to maximize their expected payoff subject to the constraint that buyers of that type get their market utility level.

Consider first a symmetric environment where $\hat{\beta}_{i}=\hat{\beta}$ for $i=1,2$. In this case, both buyer types get the same utility level $U^{*}$ in equilibrium, and all sellers get utility $V^{*}$. Figure 2 represents the indifference curves of the sellers of good 1 on the space $(k, p)$, as well as the indifference curves of the two buyer types when they trade good 1. (The situation is symmetric for good 2.) The indifference curve of type- 1 buyers is a parallel shift upwards of that of type-2 buyers, the vertical distance between the two curves being $u_{H}-u_{L}$. So it is clear from Figure 2 that, under the market utility assumption, type- $i$ sellers trade only with type- $i$ buyers. Also, all sellers choose the same price $p^{*}$ and, since beliefs are rational, this price generates market tightness $\hat{\beta} / \hat{\sigma}$ for both goods.

Consider now a more interesting environment where the proportion of type- 1 buyers is reduced, and the economy-wide market tightness level is still $\hat{\beta} / \hat{\sigma}$ (e.g. an economy with a warmer climate). If $\hat{\beta}_{1}$ is close to $\hat{\beta}$, this economy is a small perturbation of the previous one, and the equilibrium outcome still entails perfect sorting. The price of good 1 is now lower and the expected payoff of type-1 buyers (sellers) is higher (lower) reflecting the decrease in net demand, while the situation is reversed in the case of good 2. However, if $\beta_{1}$ is sufficiently low, the perfect sorting outcome is not an equilibrium. Consider such an outcome. It is easy to check that the indifference curves of type-1 buyers associated to both goods are steeper as they shift downwards (and still parallel to each other). Conversely, the indifference curves of type-2 buyers are flatter as they shift upwards. For each good then the indifference curves of type- 1 and type- 2 buyers satisfy the single crossing property. In Figure 3, $p_{1 S}$ denotes the price of good 1, $V_{1 S}$ denotes the utility obtained by sellers of good 1 , and $U_{i S}$ denotes the utility obtained by type- $i$ buyers under perfect sorting. ${ }^{26}$ (The thin curves represent the indifference curves of the traders in the equilibrium of the symmetric environment). Clearly, the perfect sorting outcome is not an equilibrium since type-2 buyers would rather trade at price $p_{1 S}$ with probability $\alpha\left(\hat{\beta}_{1} / \hat{\sigma}\right)$ obtaining a utility level higher than $U_{2 S}$.

\footnotetext{
${ }^{26}$ The corresponding graph for good 2 is the same as before, except for the shift in the agents' indifference curves.
} 
The equilibrium is depicted in Figures 4 and 5. All indifference curves shift in the same direction as in the perfect sorting outcome, but they shift by less (i.e., the changes in agents' payoffs are smaller). Figure 4 shows that a subset of type- 1 sellers choose to trade with the type- 1 buyers at price $p_{11}^{*}$, while the rest choose to trade with a subset of type-2 buyers at price $p_{12}^{*}$. Figure 5 shows that type- 2 sellers choose to trade with the remainder of the type- 2 buyers at price $p_{2}^{*}$. Note that type- 1 buyers pay a higher price for good 1 than type- 2 buyers, but they are less likely to be rationed. Also, type-2 buyers who buy good 1 pay a lower price and are less likely to be rationed than those who buy good 2. Since all these buyers (who prefer good 2) must get the same utility level, the conditions in the market of good 1 must be relatively more attractive. It remains to characterize the equilibrium beliefs in inactive submarkets (e.g. for good $h_{2}$ and prices $p_{2} \neq p_{2}^{*}$, and for good $h_{1}$ and $\left.p_{1} \notin\left\{p_{12}^{*}, p_{11}^{*}\right\}\right)$. Since it is the indifference curve of the buyer type who is most eager to trade in each inactive submarket that matters in determining these beliefs, $k_{2}^{*}\left(p_{2}\right)$ coincides with indifference curve of type-2 buyers in Figure 5 . In turn, $k_{1}^{*}\left(p_{1}\right)$ is given by the upper envelop of the indifference curves of type- 1 and type- 2 buyers in Figure 4.

Again this outcome can be decentralized as a competitive equilibrium. In Figure 5 take any continuous function $p_{2}\left(k_{2}\right)$ lying between the indifference curves of the seller and type- 2 buyers. In Figure 4 take any continuous function $p_{1}\left(k_{1}\right)$ lying between the indifference curves of the seller and the two buyer types which is tangent to the indifference curves of the seller and type- 1 buyers at $E_{11}$, and to the indifference curves of the seller and type-2 buyers at $E_{12}$. If we apply the price selection rule in Example 1, $p_{1}^{*}\left(k_{1}\right)$ and $p_{2}^{*}\left(k_{2}\right)$ are the inverses of the functions $k_{1}^{*}\left(p_{1}\right)$ and $k_{2}^{*}\left(p_{2}\right)$ which describe the equilibrium beliefs in a directed search equilibrium.

\subsection{The equivalence result}

Consider the general environment in Eeckhout and Kircher (2010). This environment is a special class of the economies studied in this paper. To facilitate comparison with our model, we use a slightly different but equivalent notation to the one used by these authors. In their model, $H=[\underline{h}, \bar{h}] \subset \Re_{+}$and $B=[\underline{b}, \bar{b}] \subset \Re_{+}$. The population is described by the measures $\xi^{\mathcal{B}} \in M_{+}(B)$ and $\xi^{\mathcal{S}} \in M_{+}(H)$. It is assumed that the c.d.f. associated to each of these measures is $C^{2}$ and the corresponding p.d.f. is strictly positive. Buyer valuations are given by a strictly positive function $u: H \times B \rightarrow \Re_{++}$. Sellers have identical valuations, normalized to zero. It is assumed that $u$ is $C^{2}$ 
with strictly positive derivatives. So, in particular, higher buyer types have higher valuations for all goods, and the higher the value of $h$ the more all buyers value a good (i.e., the higher the good's quality). The matching function $\mathcal{M}$ is strictly increasing, strictly concave and $C^{2}$, and the sellers' matching probability $\pi(k)$ has a strictly decreasing elasticity.

In a directed search equilibrium, sellers choose a price $p$ in the set of feasible prices $P \equiv$ $[0, u(\bar{b}, \bar{h})]$. Buyers choose a quality-price combination $(h, p) \in H \times P$, or they resort to autarky. The sellers' decisions are characterized by a measure $\hat{\mu}^{\mathcal{S}} \in M_{+}(H \times P)$. The buyers' decisions are characterized by a measure $\hat{\mu}^{\mathcal{B}} \in M_{+}(B \times(\{H \times P\} \cup \phi))$ where $\phi=\left(\phi_{h}, \phi_{p}\right) \in \Re_{--}^{2}$ denotes the autarky choice. All traders have rational expectations about the ratio of buyers to sellers at each quality-price combination. These expectations are described by the "queue length function" $\hat{k}: H \times P \rightarrow[0, \infty]$, which is assumed continuous. It is useful to define the restriction of $\hat{\mu}_{H \times P}^{\mathcal{B}}$ to $H \times P$, denoted by $\tilde{\hat{\mu}}_{H \times P}^{\mathcal{B}}$

Definition 2. A directed search equilibrium is a pair of measures $\left(\hat{\mu}^{\mathcal{S} *}, \hat{\mu}^{\mathcal{B} *}\right) \in M_{+}(H \times P) \times M_{+}(B \times$ $(\{H \times P\} \cup \phi))$, and a queue length function $\hat{k}^{*}: H \times P \rightarrow[0, \infty]$ such that

(i) For each type $h \in H$, sellers choose a price that maximizes their expected utility taking $\hat{k}^{*}$ as given:

$$
v_{h}^{*} \equiv \max _{p \in P} \pi\left(\hat{k}^{*}(h, p)\right) p=\pi\left(\hat{k}^{*}\left(h, p^{*}\right)\right) p^{*}, \text { for almost all }\left(h, p^{*}\right) \in \operatorname{supp} \hat{\mu}^{\mathcal{S} *}
$$

(ii) For each type $b \in B$, buyers choose a quality-price combination that maximizes their expected utility taking $\hat{k}^{*}$ as given:

$$
\hat{v}_{b}^{*} \equiv \max _{(h, p) \in\{H \times P\} \cup \phi} \alpha\left(\hat{k}^{*}(h, p)\right)[u(b, h)-p]=\alpha\left(\hat{k}^{*}\left(h^{*}, p^{*}\right)\right)\left[u\left(b, h^{*}\right)-p^{*}\right]
$$

for almost all $\left(b, h^{*}, p^{*}\right) \in \operatorname{supp} \hat{\mu}^{\mathcal{B} *}$.

(iii) $\left(\hat{\mu}^{\mathcal{B} *}, \hat{\mu}^{\mathcal{S} *}\right)$ is consistent with the population:

$$
\hat{\mu}_{B}^{\mathcal{B} *}=\xi^{\mathcal{B}} \text {, and } \hat{\mu}_{H}^{\mathcal{S} *}=\xi^{\mathcal{S}} .
$$

(iv) Beliefs are rational on the path of play, so $\tilde{\hat{\mu}}_{H \times P}^{\mathcal{B} *}$ is absolutely continuous with respect to $\hat{\mu}^{\mathcal{S} *}$ 
and, on the support $\hat{\mu}^{\mathcal{S} *}$, the corresponding Radon-Nikodym derivative is given by $\hat{k}^{*}$ :

$$
\int_{(h, p) \in \hat{F}} d \tilde{\hat{\mu}}_{H \times P}^{\mathcal{B}}(h, p)=\int_{(h, p) \in \hat{F}} \hat{k}^{*}(h, p) d \hat{\mu}^{\mathcal{S}}(h, p) \text { for all Borel } \hat{F} \subseteq H \times P .
$$

(v) Beliefs in inactive markets satisfy the market utility property:

$$
\hat{k}^{*}(h, p)=\sup \left\{k \in \Re_{+} \mid \exists b \in B \text { s.t. } \alpha(k)[u(b, h)-p]=\hat{v}_{b}^{*}\right\}
$$

if the above set is non-empty, and $\hat{k}^{*}(h, p)=0$ otherwise.

Conditions (i) and (ii) require that the traders' decisions are optimal given their beliefs. ${ }^{27}$ Conditions (iii) and (iv) are the equivalent of the consistency conditions in Definition 1. In particular, (iv) says that beliefs are rational in all active markets. In addition to the optimality and consistency conditions, here there is an additional condition which is a restriction on beliefs in inactive submarkets. Condition (v) says that either the ratio of buyers to sellers in each inactive submarket gives the buyer types who are most eager to trade there their market level of utility $\hat{v}_{b}^{*}$, or the submarket attracts no buyers at all and the ratio of buyers to sellers is zero. Below we show that this condition is the equivalent to the price selection rule we have previously described.

We begin with a preliminary result. With the aforementioned price selection rule and since the matching function is strictly increasing, a competitive equilibrium price system is strictly decreasing in $k$ for positive prices. In words, the higher the ratio of buyers to sellers the lower the price of a good. This is intuitive since buyers trade with lower probability and sellers trade with higher probability when $k$ is higher.

Proposition 1. Let $\left(\mu^{\mathcal{B} *}, \mu^{\mathcal{S} *}, \underline{p}^{*}\right)$ be a competitive equilibrium with $\underline{p}^{*}=\inf P^{*}$, where $P^{*}$ is the set of prices supporting allocation $\left(\mu^{\mathcal{B} *}, \mu^{\mathcal{S} *}\right)$. The fact that the matching function is strictly increasing implies that, for given $h \in H, \underline{p}^{*}(h, k)$ is strictly decreasing in $k$ when $\underline{p}^{*}(h, k)>0$.

We now state the equivalence result. For the sake of clarity, let us focus on equilibria where no agent type resorts to autarky. ${ }^{28}$

\footnotetext{
${ }^{27}$ Since the objective functions of buyers and sellers are continuous, and their choice sets are compact, a supremum is attained both in (i) and (ii).

${ }^{28} \mathrm{It}$ is easy to extend the equivalence result to environments where this is not the case (but the notation is messier).
} 
Proposition 2. Suppose the matching function is strictly increasing. Let $\left(\mu^{\mathcal{B} *}, \mu^{\mathcal{S} *}, \underline{p}^{*}\right)$ be a competitive equilibrium where no agent type resorts to autarky, and $\underline{p}^{*}=\inf P^{*}$ where $P^{*}$ is the set of prices supporting allocation $\left(\mu^{\mathcal{B} *}, \mu^{\mathcal{S} *}\right)$. Construct measures $\hat{\mu}^{\mathcal{S} *} \in M_{+}(H \times P)$ and $\hat{\mu}^{\mathcal{B} *} \in$ $M_{+}(B \times(\{H \times P\} \cup \phi))$ so their respective supports satisfy

$$
\begin{gathered}
(h, p) \in \operatorname{supp} \hat{\mu}^{\mathcal{S} *} \quad \text { iff } \exists k \in \Re_{+} \text {s.t. }(h, k) \in \operatorname{supp} \mu^{\mathcal{S} *} \text { and } p=p(h, k), \\
(b, h, p) \in \operatorname{supp} \hat{\mu}^{\mathcal{B} *} \quad \text { iff } \exists k \in \Re_{+} \text {s.t. }(b, h, k) \in \operatorname{supp} \mu^{\mathcal{B} *} \text { and } p=p(h, k) .
\end{gathered}
$$

Also, for all Borel $D \subseteq H, E \subseteq B$, and $G_{p} \subseteq P$,

$$
\begin{aligned}
\hat{\mu}^{\mathcal{S}}\left(D \times G_{p}\right) & =\int_{(h, k) \in F\left(D, G_{p}\right)} d \mu^{\mathcal{S} *}(h, k) \\
\hat{\mu}^{\mathcal{B} *}\left(E \times D \times G_{p}\right) & =\int_{(b, h, k) \in E \times F\left(D, G_{p}\right)} d \mu^{\mathcal{B} *}(b, h, k)
\end{aligned}
$$

where $(h, k) \in F\left(D, G_{p}\right)$ iff $h \in D$ and there is $p \in G_{p}$ with $p(h, k)=p$. Finally, define the queue length function $\hat{k}^{*}$ as follows

$$
\hat{k}^{*}(h, p)=\left\{\begin{array}{cc}
k \text { s.t. } \underline{p}^{*}(h, k)=p & \text { for } p>0 \text { in the range of } \underline{p}^{*}, \\
\inf \left\{k \in \Re_{+} \mid p(h, k)=0\right\} & \text { for } p=0, \\
0 & \text { for } p \text { outside the range of } \underline{p}^{*} .
\end{array}\right.
$$

Then $\left(\hat{\mu}^{\mathcal{S} *}, \hat{\mu}^{\mathcal{B} *}, \hat{k}^{*}\right)$ is a directed search equilibrium.

The argument in the proof shows that, given a competitive equilibrium where all agent types trade, it is direct to construct an equivalent directed search equilibrium provided the matching function is strictly increasing. In particular, for given $h, \hat{k}^{*}(h, p)$ is the inverse of $p^{*}(h, k)$ for prices $p>0$ in the range of the competitive equilibrium price system. The rest of the combinations $(h, p)$ essentially correspond to submarkets which attract no buyers so $\hat{k}^{*}(h, p)=0$.

Eeckhout and Kircher (2010) also that, in the presence of complementarities, an interesting trade-off arises between the gains from higher match values and the losses due to rationing (see also Shi 2001). They then derive conditions - which regard the degree of supermodularity of the

Think first of the economy as if the types who do not trade in a competitive equilibrium where not there. Given a competitive equilibrium, Proposition 2 constructs an equivalent directed search equilibrium by characterizing the queue length function and the measures that describe the traders' decisions. It is then trivial to incorporate the types who resort to autarky by suitably expanding those measures to include the autarky choice. 
match value function - for the equilibrium allocation to entail either positive or negative assortative matching. ${ }^{29}$ They also show that under these conditions, an equilibrium exists and is constrained efficient. Note that Theorems 2 and 4 and Proposition 2 imply the following result.

Proposition 3. The fact that matching function is strictly increasing implies that a directed search equilibrium exists and is constrained efficient in Eeckhout and Kircher (2010).

Proposition 3 thus generalizes the existence and efficiency results in Eeckhout and Kircher (2010) (which hold regardless of the matching pattern displayed by the equilibrium allocation). The only assumption we need, in addition to those in Section 2, is that $\mathcal{M}$ is strictly increasing.

In principle, many possible matching patterns could arise in equilibrium. In simple examples with two-sided heterogeneity (like the one above) the equilibrium matching pattern could be identified through trial and error. For a given matching pattern, there is a candidate feasible outcome satisfying the Hosios and the rational beliefs conditions. We just need to check whether there exist prices supporting this outcome. In more complex environments, the formulation in this paper will allow us to identify the equilibrium matching pattern and calculate the equilibrium by means of linear programming algorithms. In the Appendix we show that the equilibrium price associated to any commodity traded in equilibrium is given by the shadow price of the matching condition associated to that commodity in the planner's problem. Looking for equilibrium prices amounts to looking for shadow prices that satisfy the Complementary Slackness Theorem of linear programming. The complementary slackness conditions are then the conditions that an efficient matching pattern must satisfy in addition to feasibility.

\footnotetext{
${ }^{29}$ Specifically, the equilibrium allocation displays positive assortative matching when the match value function is $\bar{n}$-root-supermodular where $\bar{n}$ is determined by the upper bound of the elasticity of the matching function $\mathcal{M}$. It displays negative assortative matching when the match value function is nowhere $\underline{n}$-root-supermodular where $\underline{n}$ is determined by the lower bound of the elasticity of $\mathcal{M}$.
} 


\section{Appendix A}

\section{A.1 The linear programming model}

In this section, we use linear programming techniques and duality theory to prove Theorems 1,3 and 4. An alternative proof of Theorem 2 is also provided as a byproduct. We begin with some necessary notation.

Let $X$ be a locally compact separable metric space. We write $C(X)$ for the space of continuous real-valued functions on $X$, endowed with the topology of uniform convergence on compact sets. The topological dual of $C(X)$ is the space of signed regular Borel measures on $X$ with compact support (see Hewitt 1959). This space is denoted by $M_{c}(X)$, and is endowed with the weak-star topology. Then $C(X)$ is also the dual of $M_{c}(X)$. We write $C_{+}(X)$ and $M_{c+}(X)$ for the respective positive cones of these spaces.

Let $C(X)$ be paired in duality with $M_{c}(X)$ with the standard bilinear form:

$$
\langle f, \gamma\rangle=\int_{x \in X} f(x) d \gamma(x), \quad f \in C(X), \gamma \in M_{c}(X) .
$$

In the special case where $X$ is compact, the topological dual of $C(X)$ is the space of signed regular Borel measures on $X$, denoted by $M(X) .{ }^{30}$ If $X$ is finite, both $C(X)$ and $M(X)$ are isomorphic to the Euclidean space, so the integral above is replaced by a finite sum.

For any integer $n$, the product spaces $\prod_{j=1, \ldots, n} C\left(X_{j}\right)$ and $\prod_{j=1, \ldots, n} M_{c}\left(X_{j}\right)$, endowed with the corresponding product topologies, are also paired in duality with bilinear form:

$$
\sum_{j=1}^{n}\left\langle f_{j}, \gamma_{j}\right\rangle, \quad\left(f_{1}, f_{2}, \ldots, f_{n}\right) \in \prod_{j=1, \ldots, n} C\left(X_{j}\right),\left(\gamma_{1}, \gamma_{2}, \ldots, \gamma_{n}\right) \in \prod_{j=1, \ldots, n} M_{c}\left(X_{j}\right) .
$$

We denote the support of an arbitrary measure $\gamma \in M_{c}(X)$ by $\operatorname{supp} \gamma$.

In the planner's problem in Section 4, it is convenient to extend the matching condition (3.3) to include the autarky choice, and write

$$
\int_{(h, k) \in F} \alpha(k) d \mu_{H \times K}^{\mathcal{B}}(h, k)=\int_{(h, k) \in F} \pi(k) d \mu^{\mathcal{S}}(h, k) \text { for all Borel } F \subseteq H \times K,
$$

since $\pi\left(k_{0}\right)=\alpha\left(k_{0}\right)=0$. We shall use the following more compact notation though. Define the functions $\hat{\alpha}: H \times K \rightarrow[0,1]$ and $\hat{\pi}: H \times K \rightarrow[0,1)$ where

$$
\begin{aligned}
& \hat{\alpha}(h, k)=\alpha(k), \\
& \hat{\pi}(h, k)=\pi(k) .
\end{aligned}
$$

Define now the measure $\eta\left(\hat{\alpha}, \mu_{H \times K}^{\mathcal{B}}\right) \in M_{c}(H \times K)$ which is absolutely continuous with respect to

\footnotetext{
${ }^{30}$ As noted by Hewitt (1959), the topology of uniform convergence on compact sets coincides with the uniform norm topology in this case.
} 
$\mu_{H \times K}^{\mathcal{B}}$ with Radon-Nikodym derivative $\hat{\alpha}$ :

$$
d \eta\left(\hat{\alpha}, \mu_{H \times K}^{\mathcal{B}}\right)=\hat{\alpha} d \mu_{H \times K}^{\mathcal{B}} .
$$

Similarly, define $\eta\left(\hat{\pi}, \mu^{\mathcal{S}}\right) \in M_{c}(H \times K)$ where

$$
d \eta\left(\hat{\pi}, \mu^{\mathcal{S}}\right)=\hat{\pi} d \mu^{\mathcal{S}} .
$$

Condition (A.1) says that these two measures are equal.

With this equivalent description of the matching condition, the problem of the planner is to find $\left(\mu^{\mathcal{B}}, \mu^{\mathcal{S}}\right) \in M_{c}(B \times H \times K) \times M_{c}(H \times K)$ to solve

$$
(P) \quad \sup \int_{B \times H \times K} \alpha(k) u(b, h) d \mu^{\mathcal{B}}(b, h, k)-\int_{H \times K} \pi(k) v(h) d \mu^{\mathcal{S}}(h, k)
$$

s.t.

$$
\begin{aligned}
\mu_{B}^{\mathcal{B}} & =\xi^{\mathcal{B}}, \\
\mu_{H}^{\mathcal{S}} & =\xi^{\mathcal{S}} \\
\eta\left(\hat{\alpha}, \mu_{H \times K}^{\mathcal{B}}\right) & =\eta\left(\hat{\pi}, \mu^{\mathcal{S}}\right), \\
\mu^{\mathcal{B}}, \mu^{\mathcal{S}} & \geq 0 .
\end{aligned}
$$

Since the functions $u$ and $v$ describing the valuations of buyers and sellers are continuous and so is the matching function, the functions we are integrating over in the objective function are continuous. Similarly, in the constraint system (A.8), $\hat{\alpha}$ and $\hat{\pi}$ are continuous. This continuity property is key to the arguments that follow.

The objective function and the constraint systems in problem $(P)$ are linear on $\left(\mu^{\mathcal{B}}, \mu^{\mathcal{S}}\right)$. Formally, problem $(P)$ is an equality-constrained linear program (see Anderson and Nash 1987). Standard results in linear programming theory show that the dual problem for $(P)$ is also a linear program. Whereas $(P)$ is a maximization problem, the dual is a minimization problem. The primal and dual problems are related because, under certain regularity conditions, the dual variables are also the shadow prices of the primal constraints and vice versa.

Denote the respective dual variables (or Lagrange multipliers) associated with constraints (A.6) and (A.7) by $q^{\mathcal{B}} \in C(B)$ and $q^{\mathcal{S}} \in C(H)$, and that associated with constraint (A.8) by $\lambda \in C(H \times K)$. The Lagrangian associated with problem $(P)$ is

$$
\begin{aligned}
& \mathcal{L}=\int_{B \times H \times K} \alpha(k) u(b, h) d \mu^{\mathcal{B}}(b, h, k)-\int_{H \times K} \pi(k) v(h) d \mu^{\mathcal{S}}(h, k)+\int_{B} q^{\mathcal{B}}(b)\left[d \xi^{\mathcal{B}}(b)-d \mu_{B}^{\mathcal{B}}(b)\right] \\
& +\int_{H} q^{\mathcal{S}}(h)\left[d \xi^{\mathcal{S}}(h)-d \mu_{H}^{\mathcal{S}}(h)\right]+\int_{H \times K} \lambda(h, k)\left[d \eta\left(\hat{\pi}, \mu^{\mathcal{S}}\right)-d \eta\left(\hat{\alpha}, \mu_{H \times K}^{\mathcal{B}}\right)\right] .
\end{aligned}
$$


Using (A.2)-(A.5) and rearranging yields

$$
\begin{aligned}
& \mathcal{L}=\int_{B} q^{\mathcal{B}}(b) d \xi^{\mathcal{B}}(b)+\int_{H} q^{\mathcal{S}}(h) d \xi^{\mathcal{S}}(h)-\int_{B \times H \times K}\left(q^{\mathcal{B}}(b)-\hat{\alpha}(h, k)[u(b, h)-\lambda(h, k)]\right) d \mu^{\mathcal{B}}(b, h, k) \\
& -\int_{H \times K}\left(q^{\mathcal{S}}(h)-\hat{\pi}(h, k)[\lambda(h, k)-v(h)]\right) d \mu^{\mathcal{S}}(h, k),
\end{aligned}
$$

where without loss of generality $\lambda\left(h, k_{0}\right)=0$ for all $h \in H$, since $\hat{\alpha}\left(h, k_{0}\right)=\hat{\pi}\left(h, k_{0}\right)=0$.

The dual problem $(D)$ is then to find $\left(q^{\mathcal{B}}, q^{\mathcal{S}}, \lambda\right) \in C(B) \times C(H) \times C(H \times K)$ to solve ${ }^{31}$

$$
\inf \int_{B} q^{\mathcal{B}}(b) d \xi^{\mathcal{B}}(b)+\int_{H} q^{\mathcal{S}}(h) d \xi^{\mathcal{S}}(h)
$$

s.t.

$$
\begin{aligned}
q^{\mathcal{B}}(b) & \geq \hat{\alpha}(h, k)[u(b, h)-\lambda(h, k)] \quad \text { for all }(b, h, k) \in B \times H \times K, \\
q^{\mathcal{S}}(h) & \geq \hat{\pi}(h, k)[\lambda(h, k)-v(h)] \quad \text { for all }(h, k) \in H \times K .
\end{aligned}
$$

\section{A.2 Existence of optimal solutions and absence of a duality gap}

Denote the optimal values for problems $(P)$ and $(D)$ by $\nu(P)$ and $\nu(D)$, respectively. We first show that both problems are consistent (i.e. their feasible sets are not empty) and bounded (i.e. $\nu(P)$ and $\nu(D)$ are finite).

Lemma A. 1. Problems $(P)$ and $(D)$ are consistent and bounded.

Proof. Since the autarky allocation where no one trades is a feasible solution for problem $(P)$, problem $(P)$ is consistent. Also, since the gains from trade under autarky are zero, $\nu(P) \geq 0$.

In problem $(D)$, set $\lambda=\lambda_{0} \in C(H \times K)$ where $\lambda_{0}(h, k)=0$ for all $(h, k) \in H \times K$. In the constraint systems (A.10) and (A.11), $\hat{\alpha}(h, k)$ and $\pi(\hat{h}, k)$ are bounded above by one and below by zero (since they are probabilities). One then can find a feasible solution where $\lambda=\lambda_{0}$ by choosing

\footnotetext{
${ }^{31}$ Using a more compact notation, the primal problem is to find $x=\left(\mu^{\mathcal{B}}, \mu^{\mathcal{S}}\right) \in M_{c}(B \times H \times K) \times M_{c}(H \times K)$ to solve

$$
\begin{aligned}
(P) \sup & \langle x, c\rangle \\
\text { s.t. } & A x=b, \\
& x \geq 0 .
\end{aligned}
$$

Here $c=\left(c^{\mathcal{B}}, c^{\mathcal{S}}\right) \in C(B \times H \times K) \times C(H \times K)$ is given by $c^{\mathcal{B}}(b, h, k)=\hat{\alpha}(h, k) u(b, h)$ and $c^{\mathcal{S}}(h, k)=-\hat{\pi}(h, k) v(h)$, and $b=\left(\xi^{\mathcal{B}}, \xi^{\mathcal{S}}, 0\right) \in M(B) \times M(S) \times M_{c}(H \times K)$. Finally, $A: M_{c}(B \times H \times K) \times M_{c}(H \times K) \rightarrow M(B) \times M(H) \times M_{c}(H \times K)$ is a continuous linear map defined by $A\left(\mu^{\mathcal{B}}, \mu^{\mathcal{S}}\right)=\left(\mu_{B}^{\mathcal{B}}, \mu_{H}^{\mathcal{S}}, \eta\left(\hat{\alpha}, \mu_{H \times K}^{\mathcal{B}}\right)-\eta\left(\hat{\pi}, \mu^{\mathcal{S}}\right)\right)$.

The dual problem is to find $y=\left(q^{\mathcal{B}}, q^{\mathcal{S}}, \lambda\right) \in C(B) \times C(H) \times C(H \times K)$ to solve

$$
\begin{aligned}
(D) \quad \text { inf } & \langle b, y\rangle \\
\text { s.t. } & A^{*} y \geq c,
\end{aligned}
$$

where $A^{*}: C(B) \times C(H) \times C(H \times K) \rightarrow C(B \times H \times K) \times C(H \times K)$ is the adjoint of $A$. That is, $A^{*}$ is defined by the relation $\left\langle x,\left(A^{*} y\right)\right\rangle=\langle A x, y\rangle$, for all $x \in M_{c}(B \times H \times K) \times M_{c}(H \times K)$ and all $y \in C(B) \times C(H) \times C(H \times K)$.
} 
$q_{0}^{\mathcal{B}} \in C(B)$ and $q_{0}^{\mathcal{S}} \in C(H)$ constant so that

$$
\begin{aligned}
q_{0}^{\mathcal{B}}(b) & =\sup _{(b, h) \in B \times H}\{u(b, h)\}+\epsilon, \quad b \in B, \\
q_{0}^{\mathcal{S}}(h) & =\epsilon, \quad h \in H
\end{aligned}
$$

for an arbitrary (small) $\epsilon>0$. Since $u$ is continuous and $B \times H$ is compact, the above supremum, which we denote by $\overline{q_{0}^{\mathcal{B}}}$, is attained. Thus problem $(D)$ is consistent. Moreover,

$$
\nu(D) \leq \int_{B} q_{0}^{\mathcal{B}}(b) d \xi^{\mathcal{B}}(b)+\int_{H} q_{0}^{\mathcal{S}}(h) d \xi^{\mathcal{S}}(h)=\overline{q_{0}^{\mathcal{B}}} \xi^{\mathcal{B}}(B)+\epsilon \xi^{\mathcal{S}}(H)<\infty .
$$

Finally, by the weak duality theorem (Anderson and Nash 1987, Theorem 2.1), $\nu(P) \leq \nu(D)$, so the primal and dual problems are bounded:

$$
0 \leq \nu(P) \leq \nu(D) \leq \bar{q}_{0}^{\mathcal{B}} \xi^{\mathcal{B}}(B)+\epsilon \xi^{\mathcal{S}}(H)<\infty
$$

Unlike a finite linear program, a bounded infinite linear program need not have optimal solutions. Moreover, the primal and dual values need not coincide as a "positive duality gap" may occur $(\nu(P)<\nu(D))$. Below we show that the linear programs in this paper are solvable and have the same optimal value $(\nu(P)=\nu(D))$. This establishes Theorem 1 .

We first show that problem $(P)$ is solvable.

Theorem A. 1. Problem $(P)$ has optimal solutions.

Proof. The feasible set of problem $(P)$ is bounded, and the constraint map and objective function are weak-star continuous, so the result follows from Theorem 3.20 in Anderson and Nash (1987).

Next, we show that problem $(D)$ satisfies the well-known Slater regularity condition. This implies that there is no duality gap.

Theorem A. 2. There is no duality gap: $\nu(P)=\nu(D)$.

Proof. The positive cone of $C(B \times H \times K) \times C(H \times K)$ has a non-empty interior, denoted by $Y_{0}$. Also, $\left(q_{0}^{\mathcal{B}}, q_{0}^{\mathcal{S}}, \lambda_{0}\right) \in C_{+}(B) \times C_{+}(H) \times C_{+}(H \times K)$ in the proof of Lemma A.1 is a Slater point in the feasible set of problem $(D)$. Since $\nu(D)$ is finite, Theorem 3.13 in Anderson and Nash (1987) implies that $\nu(P)=\nu(D)$.

By Theorem A.2, the Complementary Slackness Theorem (Anderson and Nash 1987, Theorem 3.2) may be applied to characterize optimal solutions for problems $(P)$ and $(D)$.

Theorem A. 3. (Complementary Slackness Theorem) Feasible solutions $\left(\mu^{\mathcal{B}}, \mu^{\mathcal{S}}\right)$ and $\left(q^{\mathcal{B}}, q^{\mathcal{S}}, \lambda\right)$ for problems $(P)$ and $(D)$ are optimal if and only if they satisfy the complementary slackness conditions:

$$
\begin{aligned}
q^{\mathcal{B}}(b) & =\hat{\alpha}(h, k)[u(b, h)-\lambda(h, k)] \text { for all }(b, h, k) \in \operatorname{supp} \mu^{\mathcal{B}} \\
q^{\mathcal{S}}(h) & =\hat{\pi}(h, k)[\lambda(h, k)-v(h)] \text { for all }(h, k) \in \operatorname{supp} \mu^{\mathcal{S}} .
\end{aligned}
$$


We still need to show that problem (D) is solvable. We start with two preliminary results. Lemma A.2 shows that the set of feasible dual solutions can be taken to be bounded without loss of generality. The proof uses the fact that $u$ and $v$ are continuous, $\hat{\alpha}$ and $\hat{\pi}$ are bounded, and $B$ and $H$ are compact sets. Lemma A.3 shows that the ratio of buyers to sellers $k$ can be restricted without loss of generality to lie on a compact subset of $\Re_{+}$(e.g. to be bounded above).

Lemma A. 2. The set of feasible dual solutions can be taken to be bounded without loss of generality. In particular, feasible dual solutions satisfy $q^{\mathcal{B}}, q^{\mathcal{S}} \geq 0$. Also, we may assume that $q^{\mathcal{B}}$ and $q^{\mathcal{S}}$ are bounded above and that $\min _{h \in H} v(h) \leq \lambda(h, k) \leq \max _{(b, h) \in B \times H} u(b, h)$ for all $(h, k) \in H \times K$.

Proof. For $k=k_{0}$ the dual constraint systems (A.10)-(A.11) imply $q^{\mathcal{B}} \geq 0, q^{\mathcal{S}} \geq 0$.

For a given $(h, k) \in H \times \Re_{++}$, if an optimal primal solution satisfies $(h, k) \in \operatorname{supp} \mu^{\mathcal{S}}$ then $(\tilde{b}, h, k) \in \operatorname{supp} \mu^{\mathcal{B}}$ for some $\tilde{b} \in B$. The reason is that the restrictions of $\mu_{H \times K}^{\mathcal{B}}$ and $\mu^{\mathcal{S}}$ to $H \times \Re_{++}$ are mutually absolutely continuous measures and thus have the same support. ${ }^{32}$ By Theorem A.3, in this case, optimal dual solutions satisfy

$$
\begin{aligned}
q^{\mathcal{B}}(\tilde{b}) & =\alpha(k)[u(\tilde{b}, h)-\lambda(h, k)], \\
q^{\mathcal{S}}(h) & =\pi(k)[\lambda(h, k)-v(h)],
\end{aligned}
$$

using (A.2)-(A.3). Since $q^{\mathcal{B}}(\tilde{b}), q^{\mathcal{S}}(h) \geq 0$ and $\alpha(k), \pi(k)>0$, it follows that $v(h) \leq \lambda(h, k) \leq$ $u(\tilde{b}, h)$. Hence,

$$
0 \leq v(h) \leq \lambda(h, k) \leq \sup _{b \in B} u(b, h), \quad(h, k) \in H \times \Re_{++} .
$$

The continuity of $u$ and $v$ and the compactness of $B$ and $H$ imply that the infimum and the supremum in (A.16) are attained. Since $\lambda$ is continuous, (A.16) also holds for $(h, 0) \in \operatorname{supp} \mu^{\mathcal{S}}$. This in turn implies that the terms on right-hand side of (A.12) and (A.13) are bounded above (since matching probabilities are bounded, and, again, $u$ and $v$ are continuous and $B$ and $H$ are compact). So there is no loss of generality in assuming that $q^{\mathcal{B}}$ and $q^{\mathcal{S}}$ are bounded above.

On the other hand, if $(h, k) \notin \operatorname{supp} \mu^{\mathcal{S}}$ then $(h, k) \notin \operatorname{supp} \mu_{H \times K}^{\mathcal{B}}$. In this case, commodity $(h, k)$ is not traded, and its shadow price $\lambda(h, k)$ can be chosen arbitrarily among all the values that satisfy

$$
\begin{aligned}
q^{\mathcal{B}}(b) & \geq \alpha(k)[u(b, h)-\lambda(h, k)], \quad \forall b \in B, \\
q^{\mathcal{S}}(h) & \geq \pi(k)[\lambda(h, k)-v(h)] .
\end{aligned}
$$

In particular, we may restrict to values of $\lambda(h, k)$ satisfying (A.16). Indeed, if (A.17) and (A.18) hold for $\lambda(h, k)>u(\bar{b}, h)=\max _{b \in B} u(b, h)$, they must also hold for $\lambda(h, k)=u(\underline{b}, h)$ since $q^{\mathcal{B}} \geq 0$. Likewise, if these equations hold for $\lambda(h, k)<v(h)$ they must hold for $\lambda(h, k)=v(h)$ since $q^{\mathcal{S}} \geq 0$. Finally, since (A.16) holds for all $(h, k) \in H \times K$,

$$
\min _{h \in H} v(h) \leq \lambda(h, k) \leq \max _{(b, h) \in B \times H} u(b, h), \text { for all }(h, k) \in H \times K .
$$

\footnotetext{
${ }^{32}$ The corresponding Radon-Nikodym derivatives are $f$ and $1 / f$ where $f(h, k)=k$. This follows from equations $(2.2)$ and (3.3) since $\alpha(k), \pi(k)>0$ for all $k>0$.
} 
Lemma A. 3. There exists a sufficiently large $\bar{k} \in \Re_{+}$such that, if all the constraints which are associated with elements $k>\bar{k}$ are eliminated from problem $(D)$, the set of optimal dual solutions does not change.

Proof. Suppose the statement in Lemma A.3 were not true. Let $\left(\mu^{\mathcal{B}}, \mu^{\mathcal{S}}\right)$ be an optimal primal solution. Take an increasing sequence $\left\{k_{j}\right\} \subset \Re_{+}$with $k_{j} \rightarrow \infty$. For each $j$ there then exists $\left(h_{j}, \hat{k}_{j}\right) \in \operatorname{supp} \mu^{\mathcal{S}}$ with $\hat{k}_{j}>k_{j}$. Equivalently, $\left(b_{j}, h_{j}, \hat{k}_{j}\right) \in \operatorname{supp} \mu^{\mathcal{B}}$ for some $b_{j} \in B$, since the restrictions of $\mu^{\mathcal{S}}$ and $\mu_{H \times K}^{\mathcal{B}}$ to $H \times \Re_{++}$are mutually exclusive measures which have the same support. (If not, Theorem A.3 would imply that the dual constraints associated to all $k>k_{j}$ can be ignored without loss of generality, since they do not bind). But then the support of $\mu^{\mathcal{S}}$ contains the sequence $\left\{\left(h_{j}, \hat{k}_{j}\right)\right\}$ where $\lim \hat{k}_{j} \rightarrow \infty$, which is impossible since this support is compact by definition.

To prove the existence of optimal dual solutions we use a similar argument to that in the proof of Theorem 5.2 in Anderson and Nash (1987) on the continuous transportation problem. This is a different and yet related infinite dimensional linear program (see also Gabriel, López-Martínez, and Hernández-Lerma 2001). As in Anderson and Nash (1987), the solvability of problem (D) cannot be settled using an argument similar to that in Theorem A.1 because the space of continuous functions on a compact set is not the dual of any normed space. We follow their approach and repose problem (D) in an enlarged space which does have this property, and then appeal to the continuity of the valuation and matching functions, and the compactness of $B$ and $H$ to show that an optimal solution in the enlarged space lies in the original space.

Theorem A. 4. Problem (D) has optimal solutions.

Proof. Let us repose problem (D) with $\left(q^{\mathcal{B}}, q^{\mathcal{S}}, \lambda\right)$ in $L^{\infty}\left(\xi^{\mathcal{B}}\right) \times L^{\infty}\left(\xi^{\mathcal{S}}\right) \times L^{\infty}\left(\xi^{\mathcal{S}} \times m\right)$, where $m$ represents Lebesgue measure on $K$. (This space is the dual of $\left.L^{1}\left(\xi^{\mathcal{B}}\right) \times L^{1}\left(\xi^{\mathcal{S}}\right) \times L^{1}\left(\xi^{\mathcal{S}} \times m\right)\right)$. The new dual problem is solvable by Theorem 3.20 in Anderson and Nash (1987) since Lemma A.2 implies that its feasible set can be taken to be bounded without loss of generality.

We now show that there exists an optimal solution of this new problem where the functions $q^{\mathcal{B}}$, $q^{\mathcal{S}}$ and $\lambda$ are continuous. Suppose that $\left(q^{\mathcal{B}}, q^{\mathcal{S}}, \lambda\right)$ is optimal. Feasibility requires that

$$
\hat{\alpha}(h, k) \lambda(h, k) \geq \hat{\alpha}(h, k) u(b, h)-q^{\mathcal{B}}(b), \quad \text { for all }(b, h, k) \in B \times H \times\left(K \mid\left\{k_{0}\right\}\right),
$$

and that

$$
q^{\mathcal{S}}(h) / k+\hat{\alpha}(h, k) v(h) \geq \hat{\alpha}(h, k) \lambda(h, k), \quad \text { for all }(h, k) \in H \times\left(K \mid\left\{0, k_{0}\right\}\right),
$$

given (2.2), (A.2) and (A.3). Remember that $\lambda\left(h, k_{0}\right)=0$ for all $h \in H$. Defining $\lambda_{1} \in L^{\infty}\left(\xi^{\mathcal{S}} \times m\right)$ so

$$
\hat{\alpha}(h, k) \lambda_{1}(h, k)=\max \left\{0, \sup _{b \in B}\left\{\hat{\alpha}(h, k) u(b, h)-q^{\mathcal{B}}(b)\right\}\right\}, \quad(h, k) \in H \times\left(K \mid\left\{k_{0}\right\}\right),
$$

and $\lambda_{1}\left(h, k_{0}\right)=0$ for all $h \in H$, then yields another optimal solution $\left(q^{\mathcal{B}}, q^{\mathcal{S}}, \lambda_{1}\right)$. We now show that the restriction of $\lambda_{1}(h, k)$ to the compact set $H \times[0, \bar{k}]$ - and in fact to any compact set - is continuous. Take a sequence $\left\{b_{i}\right\}$ in $B$ such that $\hat{\alpha}(h, k) u\left(b_{i}, h\right)-q^{\mathcal{B}}\left(b_{i}\right)$ converges to $\hat{\alpha}(h, k) \lambda_{1}(h, k)$. 
Since $B \times H \times[0, \bar{k}]$ is compact, $\hat{\alpha}(h, k) u(b, h)$ is uniformly continuous on $B \times H \times[0, \bar{k}]$. For any $\epsilon>0$ there then exists $\delta$ such that

$$
\left|\hat{\alpha}(h, k) u\left(b_{i}, h\right)-\hat{\alpha}\left(h^{\prime}, k^{\prime}\right) u\left(b_{i}, h^{\prime}\right)\right|<\epsilon, \quad i=1,2, \ldots
$$

whenever $\left(h^{\prime}, k^{\prime}\right)$ lies in a $\delta$-neighborhood of $(h, k)$, and $(h, k),\left(h^{\prime}, k^{\prime}\right) \in H \times[0, \bar{k}]$. Equations (A.20) and (A.23) then imply that

$$
\hat{\alpha}\left(h^{\prime}, k^{\prime}\right) \lambda_{1}\left(h^{\prime}, k^{\prime}\right) \geq \hat{\alpha}\left(h^{\prime}, k^{\prime}\right) u\left(b_{i}, h^{\prime}\right)-q^{\mathcal{B}}\left(b_{i}\right)>\hat{\alpha}(h, k) u\left(b_{i}, h\right)-q^{\mathcal{B}}\left(b_{i}\right)-\epsilon, \quad i=1,2, \ldots
$$

Taking the limit yields

$$
\hat{\alpha}\left(h^{\prime}, k^{\prime}\right) \lambda_{1}\left(h^{\prime}, k^{\prime}\right)+\epsilon \geq \hat{\alpha}(h, k) \lambda_{1}(h, k)
$$

A symmetric argument implies that

$$
\hat{\alpha}(h, k) \lambda_{1}(h, k)+\epsilon \geq \hat{\alpha}\left(h^{\prime}, k^{\prime}\right) \lambda_{1}\left(h^{\prime}, k^{\prime}\right)
$$

for any such $\left(h^{\prime}, k^{\prime}\right)$ and $(h, k)$. Hence, by equation (A.22), the restriction of $\hat{\alpha}(h, k) \lambda_{1}(h, k)$ to $H \times[0, \bar{k}]$ is the maximum of a continuous function and a constant function, so it is continuous. Since $\hat{\alpha}$ is continuous and strictly positive on $H \times[0, \bar{k}]$, the restriction of $\lambda_{1}$ to $H \times[0, \bar{k}]$ (the quotient of two continuous functions) is continuous.

To see that $\lambda_{1}$ is continuous, take an increasing sequence of compact sets $\left\{Z_{j}\right\}$ converging to $K$; e.g. $Z_{j}=\left[0, k_{j}\right] \cup\left\{k_{0}\right\}$ with $k_{j} \uparrow \infty$. Consider the sequence of functions $\left\{f_{j}\right\}$ where $f_{j}=\chi_{Z_{j}} \lambda_{1}$, where $\chi_{Z_{j}}$ denotes the characteristic function on $Z_{j}$; so $\lambda_{1}$ and $f_{j}$ coincide on $Z_{j}$. Since $f_{j}$ is continuous on $Z_{j}$ and $\lambda_{1}=\lim _{j \rightarrow \infty} f_{j}$, it follows that $\lambda_{1}$ is continuous.

Finally, defining

$$
\begin{aligned}
q_{1}^{\mathcal{B}}(b) & =\max _{(h, k) \in H \times\left([0, \bar{k}] \cup\left\{k_{0}\right\}\right)} \hat{\alpha}(h, k)\left[u(b, h)-\lambda_{1}(h, k)\right], \\
q_{1}^{\mathcal{S}}(h) & =\max _{k \in[0, \bar{k}] \cup\left\{k_{0}\right\}} \hat{\pi}(h, k)\left[\lambda_{1}(h, k)-v(h)\right],
\end{aligned}
$$

yields yet another optimal solution $\left(q_{1}^{\mathcal{B}}, q_{1}^{\mathcal{S}}, \lambda_{1}\right)$ since, by Lemma A.3, the constraints associated with elements $k>\bar{k}$ can be ignored without loss of generality in (A.10)-(A.11). By Berge's Maximum Theorem, $q_{1}^{\mathcal{B}}$ and $q_{1}^{\mathcal{S}}$ are continuous.

\section{A.3 Welfare and Existence Theorems}

As in Gretsky, Ostroy and Zame (1992), the welfare theorems follow from the Complementary Slackness Theorem A.3. This theorem implies a direct equivalence between the optimal solutions to problems $(P)$ and $(D)$ on the one hand, and competitive equilibrium allocations, prices and indirect utilities on the other hand. The existence of a competitive equilibrium then follows from the existence of optimal solutions to problems $(P)$ and $(D)$ established in Section A.2.

Theorem A. 5. (Welfare theorems)

(I) Let $\left(\mu^{\mathcal{B} *}, \mu^{\mathcal{S} *}, p^{*}\right)$ be a competitive equilibrium. Also, define $q^{\mathcal{B} *}(b)=v_{b}^{*}\left(p^{*}\right)$ for each $b \in B$ 
and $q^{\mathcal{S} *}(h)=v_{h}^{*}\left(p^{*}\right)$ for each $h \in H$, where $v_{i}^{*}\left(p^{*}\right)$ is the equilibrium indirect utility of type-i agents. Then $\left(\mu^{\mathcal{B} *}, \mu^{\mathcal{S} *}\right)$ solves problem $(P)$, and $\left(q^{\mathcal{B} *}, q^{\mathcal{S} *}, p^{*}\right)$ solves problem $(D)$.

(II) Suppose $\left(\mu^{\mathcal{B}}, \mu^{\mathcal{S}}\right)$ and $\left(q^{\mathcal{B}}, q^{\mathcal{S}}, \lambda\right)$ are optimal solutions for problems $(P)$ and $(D)$. Then $\left(\mu^{\mathcal{B}}, \mu^{\mathcal{S}}, \lambda\right)$ is a competitive equilibrium. Moreover, $q^{\mathcal{B}}(b)$ gives the equilibrium indirect utility of type-b buyers for each $b \in B$, and $q^{\mathcal{S}}(h)$ gives the equilibrium indirect utility of type-h sellers for each $h \in H$.

Proof. (I) Let $\left(\mu^{\mathcal{B} *}, \mu^{\mathcal{S} *}, p^{*}\right)$ be a competitive equilibrium. Condition (iii) in Definition 1 is equivalent to (A.6) and (A.7), and condition (iv) is equivalent to (A.8). Also, by assumption $\left(\mu^{\mathcal{B} *}, \mu^{\mathcal{S} *}\right)$ satisfies $(\mathrm{A} .9)$. Hence, $\left(\mu^{\mathcal{B} *}, \mu^{\mathcal{S} *}\right)$ is feasible for problem $(P)$.

Define $q^{\mathcal{B} *}(b)=v_{b}^{*}\left(p^{*}\right)$ for each $b \in B$, and $q^{\mathcal{S} *}(h)=v_{s}^{*}\left(p^{*}\right)$ for each $h \in H$. Note that (5.4) and (5.3) imply that

$$
\begin{aligned}
& v_{b}^{*}\left(p^{*}\right)=\sup _{(h, k) \in \operatorname{supp} \mu_{H \times K}^{\mathcal{B} *}} \alpha(k)\left[u(b, h)-p^{*}(h, k)\right], \quad b \in B, \\
& v_{h}^{*}\left(p^{*}\right)=\sup _{k \in \operatorname{supp} \mu_{K}^{\mathcal{S} *}} \pi(k)\left[p^{*}(h, k)-v(h)\right], \quad h \in H .
\end{aligned}
$$

Since $\mu^{\mathcal{B} *}$ and $\mu^{\mathcal{S} *}$ have compact support, so do their marginals. Hence, $q^{\mathcal{B} *} \in C(B)$ and $q^{\mathcal{B} *} \in C(H)$ by Berge's Maximum Theorem. By condition (i) in Definition 1,

$$
q^{\mathcal{B} *}(b) \geq \alpha(k)\left[u(b, h)-p^{*}(h, k)\right] \text { for all }(b, h, k) \in B \times H \times K,
$$

with equality if $(b, h, k) \in \operatorname{supp} \mu^{\mathcal{B} *}$. Likewise, by condition (ii),

$$
q^{\mathcal{S} *}(h) \geq \pi(k)\left[p^{*}(h, k)-v(h)\right] \quad \text { for all }(h, k) \in H \times K,
$$

with equality if $(h, k) \in \operatorname{supp} \mu^{\mathcal{S} *}$. Remember that $\alpha\left(k_{0}\right)=\pi\left(k_{0}\right)=0$, and $p^{*}$ is normalized so $p^{*}\left(h, k_{0}\right)=0$ for all $h \in H$. Hence, (A.31)-(A.32), combined with the definitions in (A.2)-(A.3), implies that $\left(q^{\mathcal{B} *}, q^{\mathcal{S} *}, p^{*}\right)$ is feasible for problem $(D)$ and satisfies the complementary slackness conditions (A.12) and (A.13). By Theorem A.3, $\left(\mu^{\mathcal{B} *}, \mu^{\mathcal{S} *}\right)$ and $\left(q^{\mathcal{B} *}, q^{\mathcal{S} *}, p^{*}\right)$ are then optimal solutions for problems $(P)$ and $(D)$.

(II) Let $\left(\mu^{\mathcal{B}}, \mu^{\mathcal{S}}\right)$ and $\left(q^{\mathcal{B}}, q^{\mathcal{S}}, \lambda\right)$ be optimal solutions for problems $(P)$ and $(D)$. By Theorem A.3, these solutions are feasible and satisfy the complementary slackness conditions. As noted above, the primal feasibility conditions (A.6) and (A.7) are equivalent to condition (iii) in Definition 1, while (A.8) is equivalent to condition (iv). Let $p \in C(H \times K)$ be given by $p=\lambda$. Since $\lambda\left(h, k_{0}\right)=0$, $p$ satisfies our price normalization. The dual feasibility conditions (A.10)-(A.11) and associated complementary slackness conditions (A.12)-(A.13) imply that conditions (i) and (ii) hold for this choice of the price system, and $q^{\mathcal{B}}(b)$ and $q^{\mathcal{S}}(h)$ give the indirect utility of type- $b$ buyers and type- $h$ sellers at these prices. This is just the reverse of the argument in part (I). Thus $p$ decentralizes $\left(\mu^{\mathcal{B}}, \mu^{\mathcal{S}}\right)$ as a competitive equilibrium. 


\section{A.4 Proofs of Propositions 1 and 2}

Proof of Proposition 1. Part (I) of Theorem A.5 implies that $\underline{p}^{*}$ is precisely the continuous function $\lambda_{1}$ characterized in equation (A.22) in the proof of Theorem A.4. In this equation $q^{\mathcal{B}}(b)$ represents the indirect utility of type $b$ buyers in a competitive equilibrium. For given $h \in H, \hat{\alpha}(h, k)$ is positive and strictly decreasing in $k$ for $k \in \Re_{+}$. Hence, when $\lambda_{1}(h, k)>0,\left(\right.$ A.22) implies that $\lambda_{1}=$ $\sup _{b \in B}\left\{u(b, h)-\frac{q^{\mathcal{B}}(b)}{\hat{\alpha}(h, k)}\right\}$. Also, since no buyer type chooses to resort to autarky, $q^{\mathcal{B}}(b)>0$ for all $b \in B$. So $\lambda_{1}$ is strictly decreasing in $k$.

Proof of Proposition 2. According to Proposition 1, for given $h, p^{*}(h, k)$ is strictly decreasing in $k$ when prices are positive, so its inverse is given by $\hat{k}^{*}(h, p)$ on this range (see equation (5.12)). As noted in the proof of Proposition 1, $\underline{p}^{*}$ is characterized in equation (A.22). Since $p^{*}$ is continuous, Proposition 1 also implies the following. For a given $h$, there exists $k_{0}(h)$ such that $\underline{p}^{*}(h, k)=0$ and $\sup _{b \in B}\left\{u\left(b, h^{*}\right)-v_{b}^{*}\left(p^{*}\right) / \alpha(k)\right\} \leq 0$ for all $k \geq k_{0}(h)$ with strict equality only if $k=k_{0}(h)$ and $\underline{p}^{*}(h, k)>0$ for some $k \in \Re_{+}$(see also the proof of Proposition 1). So, for a given $h, p(h, k)$ is not invertible for $k \geq k_{0}(h)$. In (5.12) we set $\hat{k}^{*}(h, 0)=\inf \left\{k \in \Re_{+} \mid p(h, k)=0\right\}=k_{0}(h)$. On the other hand, (5.8) implies that $(h, 0) \notin \operatorname{supp} \hat{\mu}^{\mathcal{S} *}$ for all $h \in H$ since all seller types trade in a competitive equilibrium. (For a seller, choosing $(h, 0)$ would be equivalent to choosing not to trade). Given all the above, equations (5.8) and (5.9) directly imply that conditions (i) and (ii) in Definition 2 hold because so do conditions (i) and (ii) in Definition 1. Moreover, in the former conditions, $\hat{v}_{i}^{*}=v_{i}^{*}\left(\underline{p}^{*}\right)$ for all $i \in B \cup H$. Note also that the measures described in (5.8)-(5.11) are well-defined.

Lemma A.2 and Part (I) of Theorem A.5 imply that, in the environment in Eeckhout and Kircher (2010), $0 \leq \underline{p}^{*}(h, k) \leq u(\bar{b}, \bar{h})$ for all $(h, k) \in H \times K$ (see equation (A.19)). So the range of $p^{*}$ is contained in the set $P^{*}$. But then (5.10) and (5.11) directly imply that conditions (iii) and (iv) in Definition 2 hold because so do conditions (iii) and (iv) in Definition 1. Note also that the range of $\underline{p}^{*}$ contains points arbitrarily close to $p=0$ (by Proposition 1).

To see that condition (v) also holds, let us go back to equation (A.22). The supremum in the righthand side of (A.22) is attained. For commodities $(h, k) \notin \operatorname{supp} \mu^{\mathcal{S} *}$ with $\underline{p}^{*}(h, k)>0$, (A.22) says that $\underline{p}^{*}(h, k)$ that gives the buyer type who is willing to pay a higher price for $(h, k)$ her equilibrium level of utility. In turn condition (v) in Definition 2 says that for submarkets $(h, p) \notin \operatorname{supp} \hat{\mu}^{\mathcal{S} *}, \hat{k}^{*}(h, p)$ gives the buyer type who is willing to accept a higher $k$ in submarket $(h, p)$ her equilibrium level of utility. Or, if this is not possible, $\hat{k}^{*}(h, p)=0$. Since the buyers' payoff is decreasing in both $p$ and $k$, Proposition 1 implies that, for given $h \in H$, the inverse of $\underline{p}^{*}(h, k)$ satisfies condition (v) when $p>0$. In sum, for $p>0$ on the range of $\underline{p}^{*}$, condition (v) holds because $\underline{p}^{*}$ satisfies (A.22). For $(h, k) \notin \operatorname{supp} \mu^{\mathcal{S} *}$ with $\underline{p}^{*}(h, k)=0$, there are two cases. Either (a) $\sup _{b \in B}\left\{u\left(b, h^{*}\right)-v_{b}^{*}\left(\underline{p}^{*}\right) / \alpha(k(h))\right\}=0$ where $k(h)$ is defined above, or (b) $\sup _{b \in B}\left\{u\left(b, h^{*}\right)-\right.$ $\left.v_{b}^{*}\left(\underline{p}^{*}\right) / \alpha(k)\right\}<0$ for all $k \in \Re_{+}$and $k(h)=0$. In case (a), the same argument used for commodities with positive prices implies that $\hat{k}^{*}(h, 0)$ satisfies condition $(\mathrm{v})$. In case (b), $(h, 0)$ attracts no buyers and so $\hat{k}^{*}(h, 0)=0$ also satisfies condition (v). It remains to deal with submarkets $(h, p)$ where $p>0$ lies outside the range of $p$. These are prices so high that they are not observed in a competitive equilibrium. Equation (A.22) implies that these submarkets attract no buyers, so $\hat{k}^{*}(h, p)=0$ also satisfies condition $(\mathrm{v})$. 


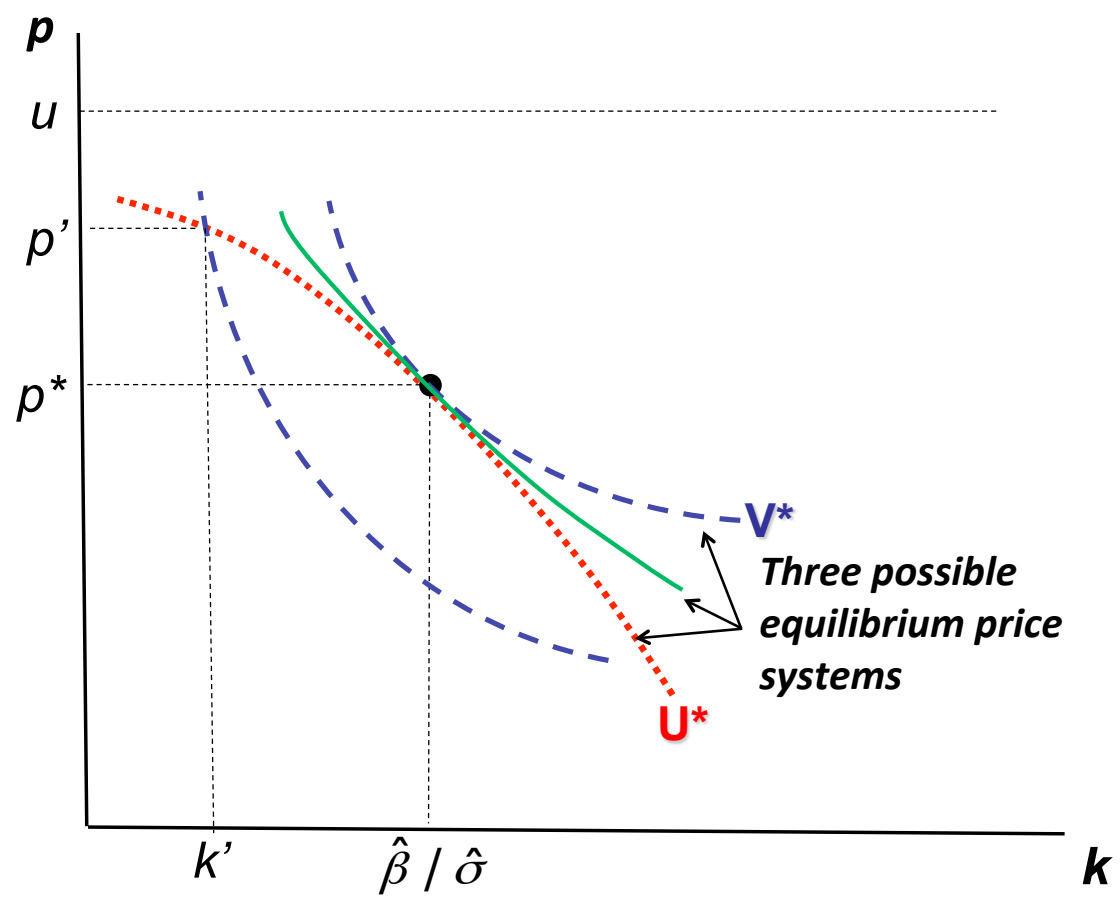

Figure 1: Homogeneous buyers and sellers.

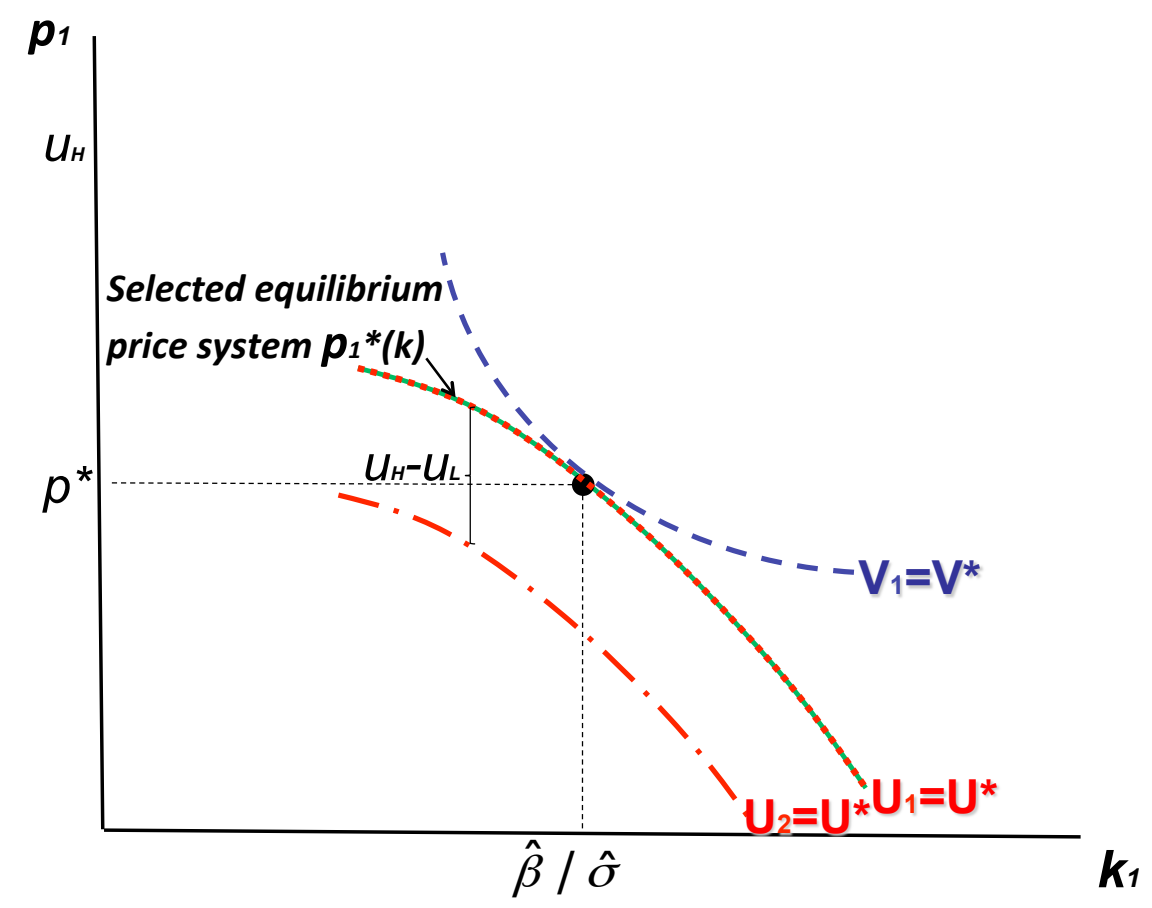

Figure 2: Heterogenous buyers and sellers (symmetric case). 


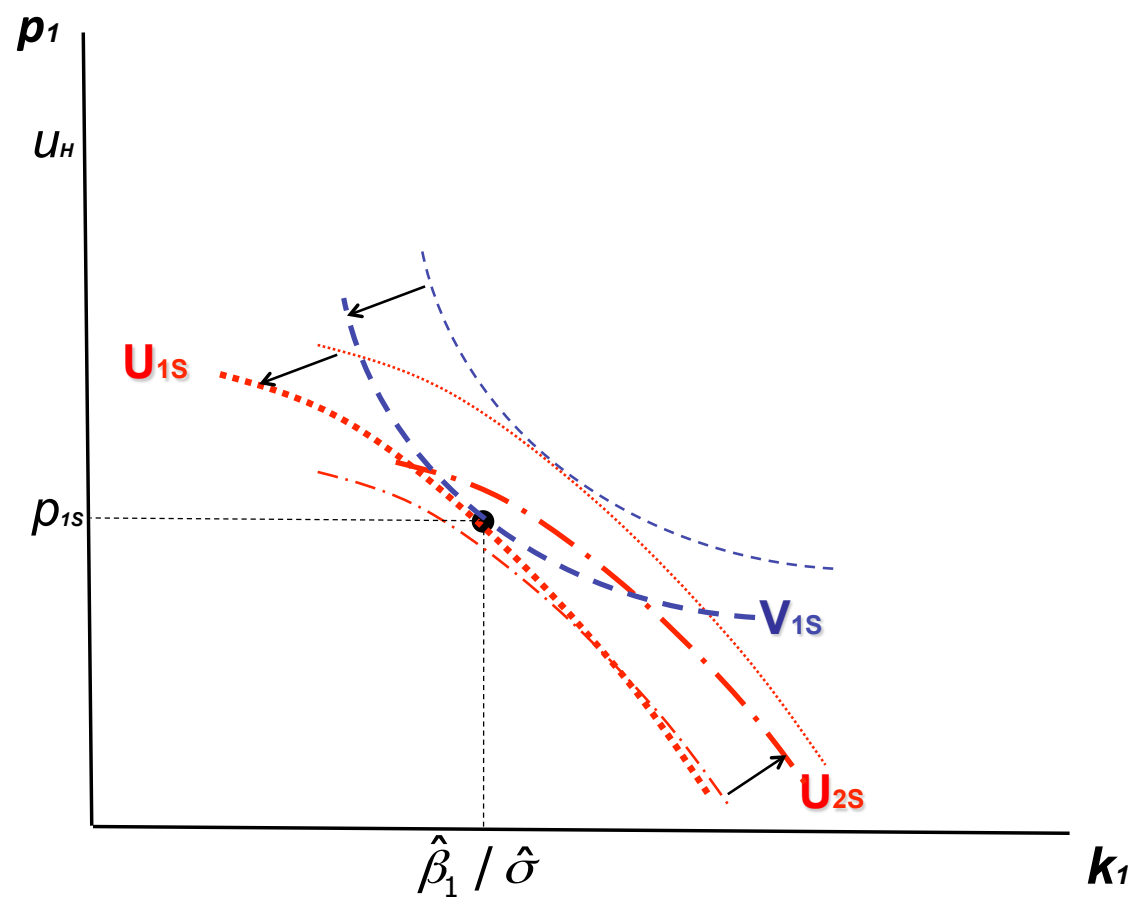

Figure 3: When perfect sorting is not an equilibrium (good 1).

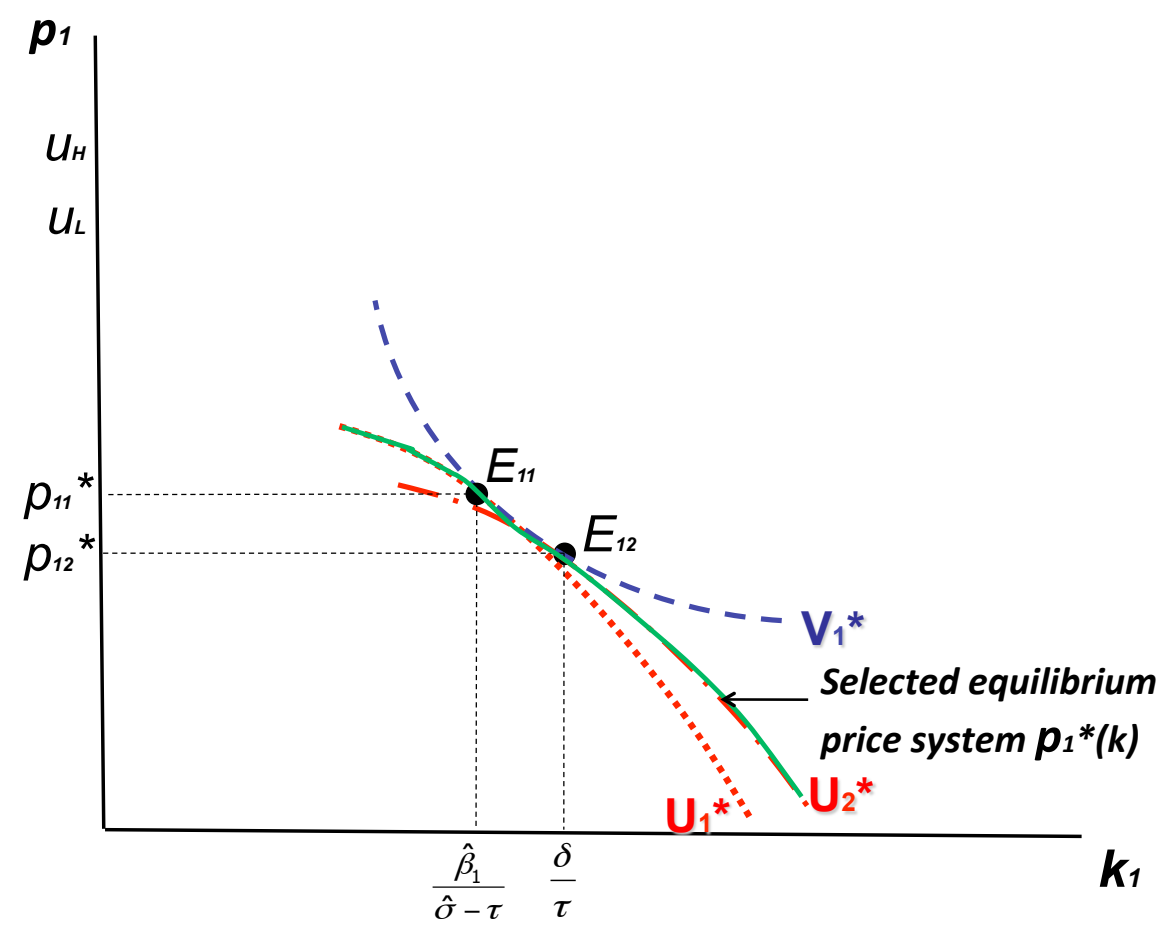

Figure 4: Equilibrium with heterogenous buyers and sellers (good 1). 


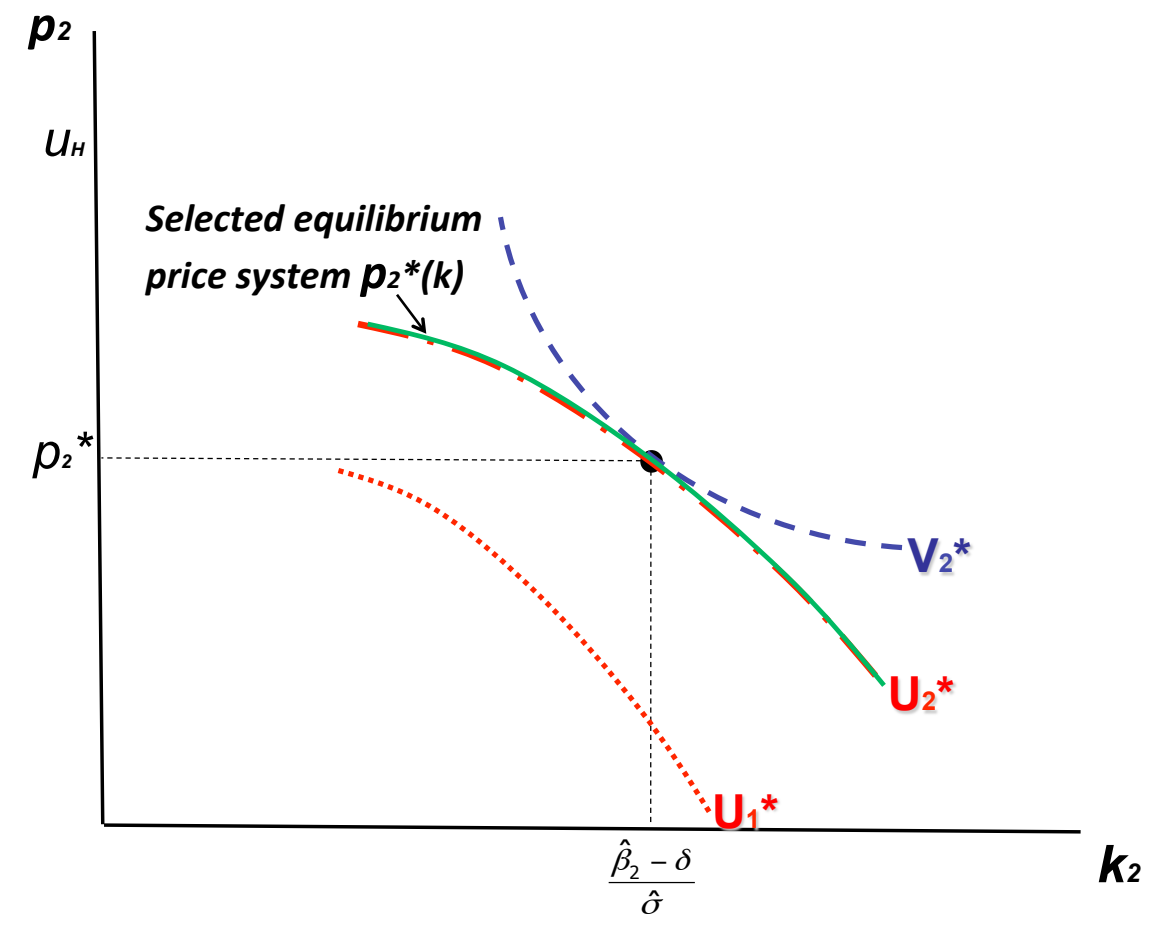

Figure 5: Equilibrium with heterogenous buyers and sellers (good 2). 


\section{References}

Acemoglu, D. and R. Shimer (1999a). Efficient unemployment insurance. Journal of Political Economy 107(5), 893-928.

Acemoglu, D. and R. Shimer (1999b). Holdups and efficiency with search frictions. International Economic Review 40(4), 827-49.

Anderson, E. J. and P. Nash (1987). Linear Programming in Infinite-Dimensional Spaces. Wiley.

Arrow, K. J. (1964). The role of securities in the optimal allocation of risk-bearing. The Review of Economic Studies 31(2), 91-96.

Burdett, K., S. Shi, and R. Wright (2001). Pricing and matching with frictions. Journal of Political Economy 109(5), 1060-1085.

Debreu, G. (1959). The Theory of Value: An Axiomatic Analysis of Economic Equilibrium. Cowles Foundation, New Haven.

Diamond, P. A. (1981). Mobility costs, frictional unemployment, and efficiency. Journal of Political Economy 89(4), 798-812.

Diamond, P. A. (1982). Wage determination and efficiency in search equilibrium. Review of Economic Studies 49(2), 217-27.

Eeckhout, J. and P. Kircher (2008). Sorting and decentralized price competition. PIER Working Paper Archive 08-020, Penn Institute for Economic Research, Department of Economics, University of Pennsylvania.

Eeckhout, J. and P. Kircher (2010). Sorting and decentralized price competition. Econometrica $78(2), 539-574$.

Ekeland, I. (2010). Existence, uniqueness and efficiency of equilibrium in hedonic markets with multidimensional types. Economic Theory 42(2), 275-315.

Ekeland, I., J. J. Heckman, and L. Nesheim (2004). Identification and estimation of hedonic models. Journal of Political Economy 112(S1), S60-S109.

Gabriel, J. R., R. R. López-Martínez, and O. Hernández-Lerma (2001). The Lagrange approach to infinite linear programs. TOP 9(2), 293-314.

Gale, D. (1996). Equilibria and Pareto optima of markets with adverse selection. Economic Theory 7(2), 207-235.

Gretsky, N. E., J. M. Ostroy, and W. R. Zame (1992). The nonatomic assignment model. Economic Theory 2(1), 103-27.

Gretsky, N. E., J. M. Ostroy, and W. R. Zame (1999). Perfect competition in the continuous assignment model. Journal of Economic Theory 88(1), 60-118.

Gretsky, N. E., J. M. Ostroy, and W. R. Zame (2002). Subdifferentiability and the duality gap. Positivity 6, 261-274.

Guerrieri, V., R. Shimer, and R. Wright (2010). Adverse selection in competitive search equilibrium. Econometrica 78(6), 1823-1862.

Hart, S., W. Hildenbrand, and E. Kohlberg (1974). On equilibrium allocations as distributions on the commodity space. Journal of Mathematical Economics 1(2), 159-166. 
Hewitt, E. (1959). Linear functionals on spaces of continuous functions. Fundamentae Mathematicae 37, 161-189.

Hosios, A. J. (1990). On the efficiency of matching and related models of search and unemployment. Review of Economic Studies 57(2), 279-98.

Jerez, B. (2003). A dual characterization of incentive efficiency. Journal of Economic Theory 112(1), 1-34.

Jerez, B. (2010). Competitive equilibrium with search frictions: Arrow-Debreu meets DiamondMortensen-Pisarides. Economics Working Papers we1039, Universidad Carlos III.

Makowski, L. and J. M. Ostroy (1996). Perfect competition via linear programming. Mimeo, UCLA.

Makowski, L. and J. M. Ostroy (2003). Competitive contractual pricing with transparent teams. Mimeo.

Mas-Colell, A. (1975). A model of equilibrium with differentiated commodities. Journal of Mathematical Economics 2(2), 263-295.

Mas-Colell, A. and W. R. Zame (1991). Equilibrium theory in infinite dimensional spaces. In W. Hildenbrand and H. Sonnenschein (Eds.), Handbook of Mathematical Economics, Volume 4 of Handbook of Mathematical Economics. Elsevier.

Menzio, G. and S. Shi (2009). Efficient search on the job and the business cycle. NBER Working Papers 14905, National Bureau of Economic Research, Inc.

Menzio, G. and S. Shi (2010). Directed search on the job, heterogeneity, and aggregate fluctuations. American Economic Review 100(2), 327-32.

Moen, E. R. (1997). Competitive search equilibrium. Journal of Political Economy 105(2), 385411.

Moen, E. R. and Å. Rosen (2011). Incentives in competitive search equilibrium. Review of Economic Studies, doi: 10.1093/restud/rdq011.

Montgomery, J. D. (1991). Equilibrium wage dispersion and interindustry wage differentials. The Quarterly Journal of Economics 106(1), 163-179.

Mortensen, D. T. (1982a). The matching process as a noncooperative bargaining game. In The Economics of Information and Uncertainty, NBER Chapters. National Bureau of Economic Research, Inc.

Mortensen, D. T. (1982b). Property rights and efficiency in mating, racing, and related games. American Economic Review 72(5), 968-79.

Mortensen, D. T. and R. Wright (2002). Competitive pricing and efficiency in search equilibrium. International Economic Review 43(1), 1-20.

Peters, M. (1991). Ex ante price offers in matching games non-steady states. Econometrica 59(5), $1425-54$.

Peters, M. (1997). On the equivalence of Walrasian and Non-Walrasian equilibria in contract markets: The case of complete contracts. Review of Economic Studies 64(2), 241-64.

Peters, M. (2000). Limits of exact equilibria for capacity constrained sellers with costly search. Journal of Economic Theory 95(2), 139-168. 
Pissarides, C. A. (1984). Search intensity, job advertising, and efficiency. Journal of Labor Economics 2(1), 128-43.

Pissarides, C. A. (1985). Short-run equilibrium dynamics of unemployment vacancies, and real wages. American Economic Review 75(4), 676-90.

Rahman, D. M. (2005). Team Formation and Organization. Ph. D. thesis, University of California, Los Angeles.

Rogerson, R., R. Shimer, and R. Wright (2005). Search-theoretic models of the labor market: A survey. Journal of Economic Literature 43(4), 959-988.

Shapley, L. S. (1955). Markets as cooperative games. Technical Report P-B29, Rand Cooporation.

Shapley, L. S. and M. Shubik (1972). The assignment game i: the core. International Journal of Game Theory 1, 111-130.

Shi, S. (2001). Frictional assignment. i. efficiency. Journal of Economic Theory 98(2), 232-260.

Shi, S. (2009). Directed search for equilibrium wage-tenure contracts. Econometrica 77(2), 561584.

Shimer, R. (1996). Contracts in a frictional labor market. Mimeo.

Shimer, R. (2005). The assignment of workers to jobs in an economy with coordination frictions. Journal of Political Economy 113(5), 996-1025.

Song, J. (2012). Futures market: contractual arrangement to restrain moral hazard in teams. Economic Theory 51(1), 163-189. 